\title{
DACH1 protects podocytes from experimental diabetic injury and modulates PTIP-H3K4Me3 activity
}

\author{
Aili Cao, ${ }^{1,2}$ Jianhua Li, ${ }^{1}$ Morad Asadi, ${ }^{1}$ John M. Basgen, ${ }^{3}$ Bingbing Zhu, ${ }^{1,2}$ Zhengzi Yi, ${ }^{1}$ Song Jiang, ${ }^{4}$ Tomohito Doke, ${ }^{5}$ Osama El Shamy, \\ Niralee Patel, ${ }^{1}$ Paolo Cravedi, ${ }^{1}$ Evren U. Azeloglu, ${ }^{1}$ Kirk N. Campbell, ${ }^{1}$ Madhav Menon, ${ }^{1}$ Steve Coca, ${ }^{1}$ Weijia Zhang, ${ }^{1}$ Hao Wang, ${ }^{2}$ \\ Ke Zen, ${ }^{4}$ Zhihong Liu, ${ }^{4}$ Barbara Murphy, ${ }^{1}$ John C. He, ${ }^{1}$ Vivette D. D’Agati, ${ }^{6}$ Katalin Susztak, ${ }^{5}$ and Lewis Kaufman ${ }^{1}$

\begin{abstract}
'Division of Nephrology, Icahn School of Medicine at Mount Sinai, New York, New York, USA. ²Department of Nephrology, Putuo Hospital, Shanghai University of Traditional Chinese Medicine, Shanghai, China. ${ }^{3}$ Life Science Institute, Charles R. Drew University of Medicine and Science, Los Angeles, California, USA. ${ }^{4}$ National Clinical Research Center of Kidney Diseases, Jinling Hospital, Nanjing University School of Medicine, Nanjing, Jiangsu, China. ${ }^{5}$ Renal Electrolyte and Hypertension Division, Perelman School of Medicine at University of Pennsylvania, Philadelphia, Pennsylvania, USA. ${ }^{6}$ Department of Pathology, Columbia University Medical Center, New York, New York, USA.
\end{abstract}

\begin{abstract}
Dachshund homolog 1 (DACH1), a key cell-fate determinant, regulates transcription by DNA sequence-specific binding. We identified diminished Dach1 expression in a large-scale screen for mutations that convert injury-resistant podocytes into injury-susceptible podocytes. In diabetic kidney disease (DKD) patients, podocyte DACH1 expression levels are diminished, a condition that strongly correlates with poor clinical outcomes. Global Dach1 KO mice manifest renal hypoplasia and die perinatally. Podocyte-specific Dach1 KO mice, however, maintain normal glomerular architecture at baseline, but rapidly exhibit podocyte injury after diabetes onset. Furthermore, podocyte-specific augmentation of DACH1 expression in mice protects from DKD. Combined RNA sequencing and in silico promoter analysis reveal conversely overlapping glomerular transcriptomic signatures between podocyte-specific Dach1 and Pax transactivation-domain interacting protein (Ptip) KO mice, with upregulated genes possessing higher-than-expected numbers of promoter Dach1-binding sites. PTIP, an essential component of the activating histone $\mathrm{H} 3$ lysine 4 trimethylation (H3K4Me3) complex, interacts with DACH1 and is recruited by DACH1 to its promoter-binding sites. DACH1-PTIP recruitment represses transcription and reduces promoter H3K4Me3 levels. DACH1 knockdown in podocytes combined with hyperglycemia triggers target gene upregulation and increases promoter H3K4Me3. These findings reveal that in DKD, diminished DACH1 expression enhances podocyte injury vulnerability via epigenetic derepression of its target genes.
\end{abstract}

\section{Introduction}

Dachshund homolog 1 (DACH1) was originally identified as an essential member of the retinal determination gene network (RDGN), a fundamental signal that governs cell fate during development. That DACH1 regulates crucial developmental functions is reinforced by the early lethality of Dach1 global homozygous null mice, although no organ morphological or metabolic abnormalities were previously detected in these mice (1). DACH1 is also widely expressed in normal adult tissues, including in kidney, where it is robustly expressed in the podocyte nucleus. It is well described as an antioncogenic protein whose reduced expression tightly correlates with poor prognosis in multiple cancers $(2,3)$. DACH1 protein regulates target gene expression either by directly binding to specific DNA sequences in chromatin $(4,5)$ or indirectly as a cointegrator by combining with other transcription factors (6-9). The DNA sequence that specifically mediates direct DACH1 DNA-specific binding has been identified (4), and the

Conflict of interest: The authors have declared that no conflict of interest exists. Copyright: () 2021, American Society for Clinical Investigation.

Submitted: June 16, 2020; Accepted: March 23, 2021; Published: May 17, 2021

Reference information: J Clin Invest. 2021;131(10):e141279.

https://doi.org/10.1172/JCl141279. result of such DNA-specific binding is transcriptional repression of the target gene $(4,5)$. Podocyte DACH1 expression is reduced in human glomerulopathy, including diabetic kidney disease (DKD) $(10,11)$, and DACH1 has been shown to be important in zebrafish nephrocyte development (11). A large genome-wide association study identified a DACH1 intronic SNP as protective of incident and prevalent human chronic kidney disease (CKD) $(12,13)$, and $D A C H 1$ was recently identified as high-yield kidney candidate gene for CKD therapeutic intervention (14). However, the role of $\mathrm{DACH} 1$ in podocytes in normal and diseased conditions remains largely unknown.

DKD is by far the leading cause of end-stage renal disease (ESRD) worldwide (15). Although therapeutic advances have dramatically decreased the rates of diabetes-related cardiovascular complications, the rates of DKD and ESRD have continued to increase (16), and there are no podocyte-targeted therapies. Previously, we identified Dach1 in a large-scale mutagenic screen as one of only a few mutations that could make genetically injury-resistant podocytes highly injury susceptible (17). In the current work, we investigate whether diminished expression of podocyte DACH1, as occurs in human DKD, plays an important role in DKD pathogenesis. We found that podocyte-specific Dach1 KO mice maintain normal glomerular architecture at baseline, but mani- 

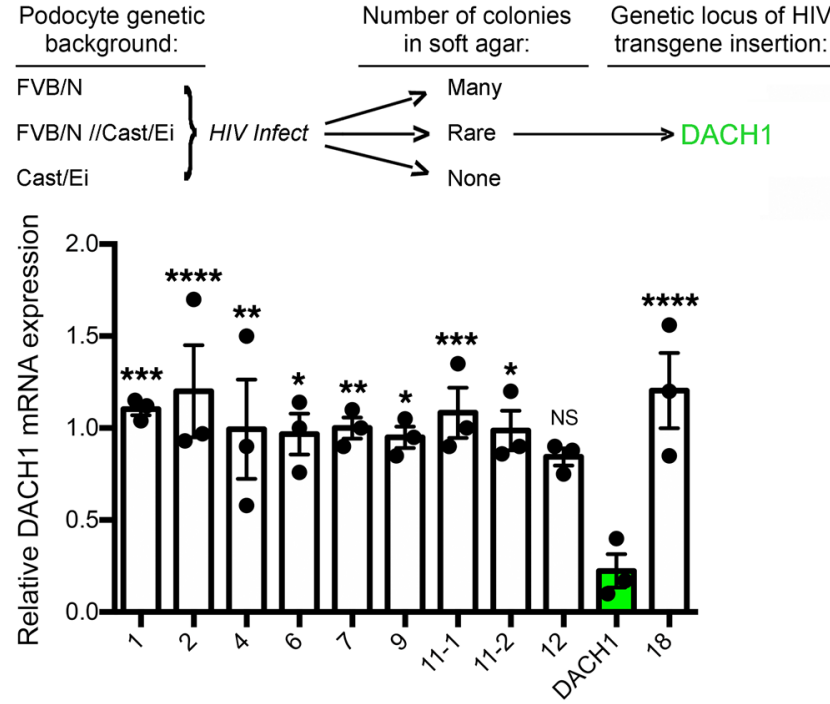

Agar clone (by chromosome of HIV insertion)
Figure 1. Diminished DACH1 expression identified as rescuing podocyte injury susceptibility. (A) Schematic of large-scale mutagenic screen (17). Immortalized podocyte cell lines from different genetic backgrounds were generated, infected with HIV provirus, and grown in soft agar. Podocytes derived from CAST/Ei mice, a background completely resistant to HIVinduced injury, did not show anchorage-independent growth, whereas cells derived from the sensitive $\mathrm{FVB} / \mathrm{N}$ background grew robustly. Podocytes from FVB×CAST F1 mice formed rare colonies, a result of HIV proviral integration influencing a host gene. The HIV integration sites of these clones were mapped, and the candidate gene Dach1 identified. (B) The podocyte clone where HIV had integrated into the Dach1 locus showed reduced Dach1 expression by qPCR. ${ }^{*} P<0.05 ;{ }^{* *} P<0.04 ;{ }^{* *} P<0.02$; ${ }^{* * *} P<0.005$. (B), 1-way ANOVA and Tukey's post hoc test. fest massive podocyte injury with rapid progression to ESRD after the onset of diabetes. Furthermore, preventing loss of podocyte DACH1 expression using podocyte-specific inducible Dach1 transgenic mice substantially ameliorates glomerular injury in OVE26 diabetic mice. These findings implicate loss of podocyte DACH1 expression as a driver of glomerular injury in DKD.

To investigate the involved mechanisms, we combined transcriptomic with in silico promoter analysis, the results of which reveal similarities between the target genes of DACH1 and those of Pax transactivation-domain interacting protein (PTIP) in podocytes. PTIP is encoded by the PAXIP1 gene and is an essential component of the MLL3/4 histone H3 lysine 4 (H3K4) methyltransferase complex $(18,19)$, an epigenetic modification associated with increased gene expression. Mounting evidence suggests that aberrant epigenetic alterations play a crucial role in DKD pathogenesis $(20,21)$ and in modifying the podocyte injury response $(22,23)$. PTIP does not possess methyltransferase activity itself, but instead functions to recruit the entire complex to specific genomic DNA-binding domains in the promoters of target genes (24). In the current work, we show that DNA sequence-specific binding of DACH1 to its promoter recruits PTIP and that this results in transcriptional repression with reduced $\mathrm{H} 3 \mathrm{~K} 4$ trimethylation (H3K4Me3) levels. In DKD, where podocyte DACH1 expression is dramatically diminished, DACH1-PTIP promoter binding is reduced, transcriptional repression is lost, and levels of $\mathrm{H} 3 \mathrm{~K} 4 \mathrm{Me} 3$ increase. Together, these findings demonstrate that in DKD, diminished DACH1 expression intensifies podocyte injury vulnerability via epigenetic derepression of multiple DACH1 target genes.

\section{Results}

Diminished DACH1 expression identified as a major susceptibility factor for podocyte injury. Previously, we performed a large-scale insertional mutagenic screen of genetically injury-resistant podocytes isolated from mice to identify genetic alterations that could rescue injury susceptibility (17) (Figure 1 $\mathrm{A}$ and $\mathrm{B})$. The readout for the screen was the ability of podo- cytes to proliferate in soft agar after HIV infection, a phenotype that is highly dependent on genetic background $(25,26)$. A mutation near the Dach1 locus that resulted in decreased Dach1 mRNA expression (Figure 1B) was one of only a few total mutations that allowed heterozygous (FVB/N;Cast/Ei) podocytes that were completely resistant to HIV-induced anchorage-independent growth at baseline to form colonies. This finding was consistent with DACH1's well-established role as an anti-oncogenic protein $(2,3)$.

In human DKD patients, podocyte DACH1 expression levels are diminished, a condition that strongly correlates with poor clinical outcomes. To investigate expression levels of podocyte DACH1 in human glomerular disease, we analyzed glomerular transcriptomic data sets available on Nephroseq and found DACH1 mRNA expression to be decreased in several glomerular diseases. This decrease was most severe in human DKD (Figure 2A), where its diminished expression outpaced that of almost all other podocyte genes (Figure 2B). DACH1 glomerular mRNA expression levels were also reduced in several forms of human primary nephrotic syndrome (27), although to a lesser extent than in DKD patients (Supplemental Figure 1; supplemental material available online with this article; https://doi.org/10.1172/JCI141279DS1). To validate the findings in a separate group of DKD patients, we performed immunofluorescence (IF) analysis for DACH1 protein on frozen kidney sections of DKD compared with control patients. Intensity of glomerular DACH1 staining was substantially diminished in DKD patients (Figure 2C). These findings were quantified using MetaMorph software to analyze fluorescent intensity per area and then normalized to DAPI intensity (Figure 2D).

To correlate levels of glomerular DACH1 mRNA expression with outcomes in human DKD patients, we studied previously reported glomerular transcriptomic data sets collected from human DKD patients (Figure 2, E and F) (28). The analysis revealed $D A C H 1$ to be one of the podocyte genes with the strongest correlations to both baseline proteinuria (correlation coefficient, $-0.71 ; P=5.20 \times 10^{-10}$ ) and EGFR (correlation coefficient, $\left.0.63 ; P=1.27 \times 10^{-07}\right) . D A C H 1$ was identified as a "hub" gene 
A

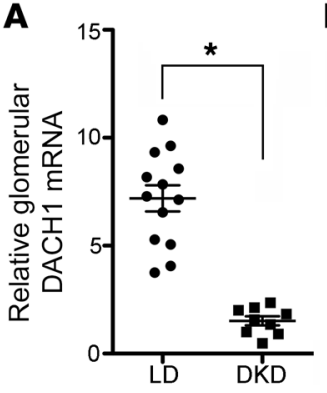

C

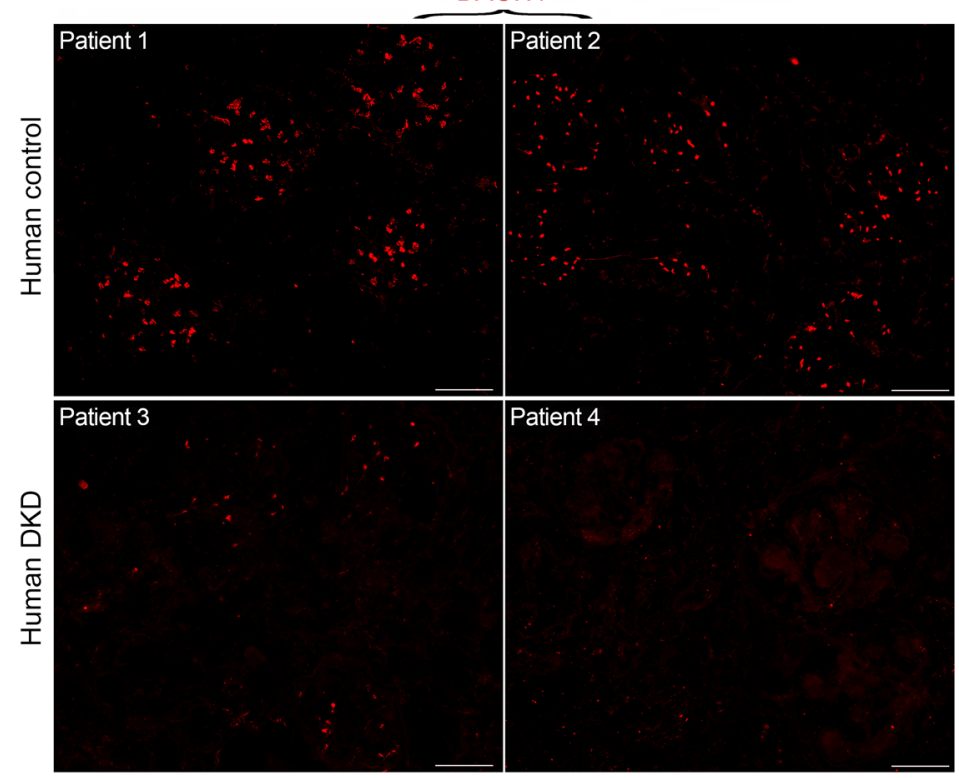

B

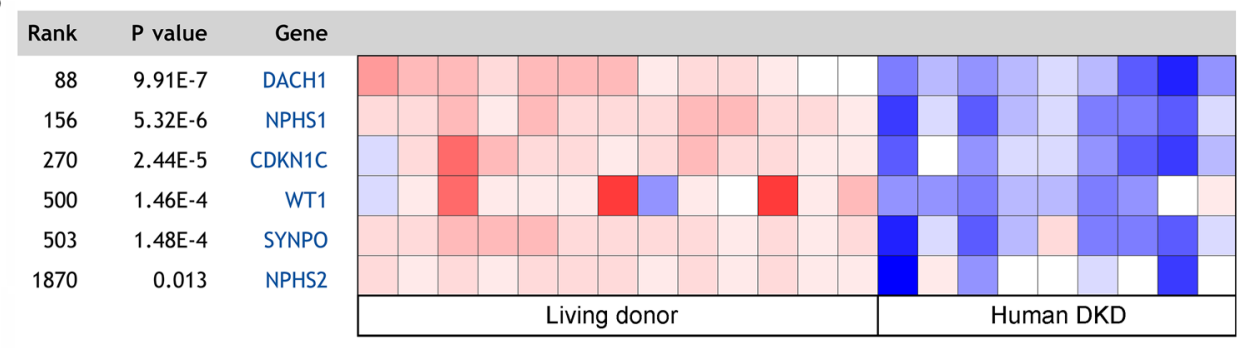

D

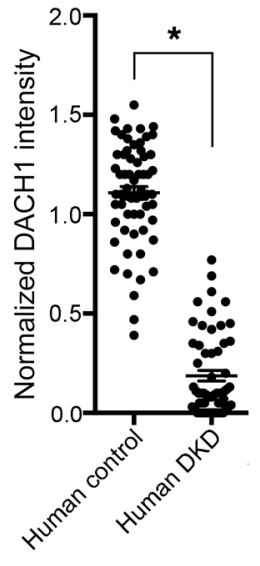

E

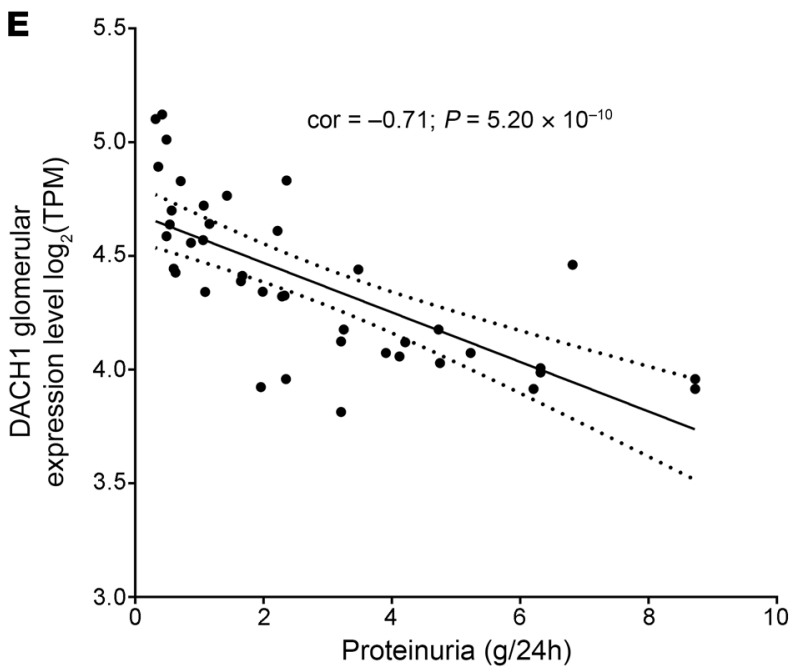

$\mathbf{F}$

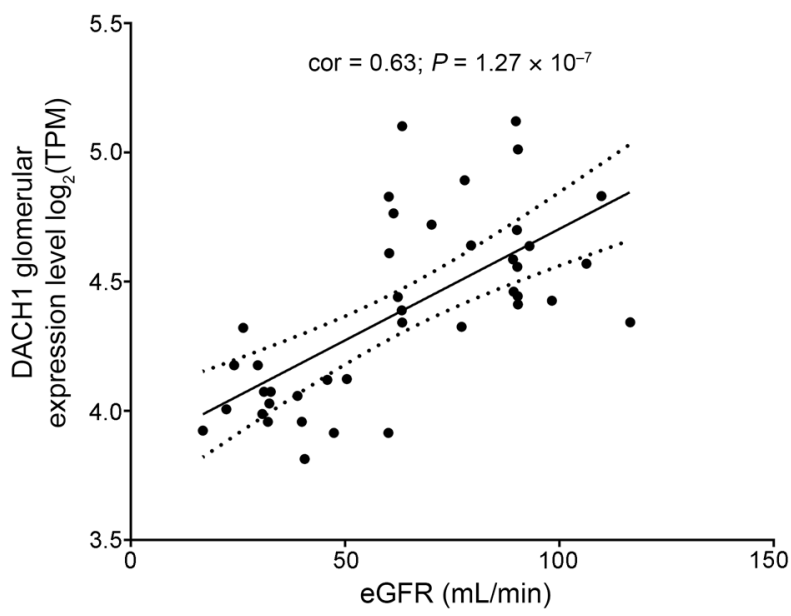

Figure 2. In human DKD patients, levels of podocyte DACH1 expression are reduced and correlate strongly with poor clinical outcomes. (A) Clomerular DACH1 mRNA expression levels are decreased in DKD patients compared with healthy living kidney donors. Data are from a previously published microarray study by Woroniecka et al. (40) and were subjected to further analysis by Nephroseq (Compendia Bioscience). ${ }^{*} P<9.91 \times 10^{-7}$ 2-tailed Student's $t$ test. (B) The magnitude of diminished glomerular DACH1 mRNA expression was in the top 1\% overall (40), higher than almost all other major podocyte genes. Adapted from Nephroseq. (C) DACH1 fluorescent staining intensity in glomeruli is diminished in human nephrectomy samples with a clinicopathological diagnosis of DKD compared with controls. Representative images taken with identical exposure times are shown. Scale bars: $100 \mu \mathrm{m}$. (D) Differences in DACH1 glomerular fluorescent intensity between DKD ( $n=4$ patients, 58 glomeruli analyzed) and controls ( $n=4$ patients, 62 glomeruli analyzed) were quantified relative to DAPI. ${ }^{*} P<0.0001$, 2-tailed Student's $t$ test. (E) Correlation between glomerular DACH1 mRNA expression levels on microarray and proteinuria $\left(n=41\right.$, correlation $\left.=-0.71, P=5.20 \times 10^{-10}\right)$. (F) Correlation blot as in E but to EGFR $\left(n=41\right.$, correlation $\left.=0.63, P=1.27 \times 10^{-07}\right)$. 
A

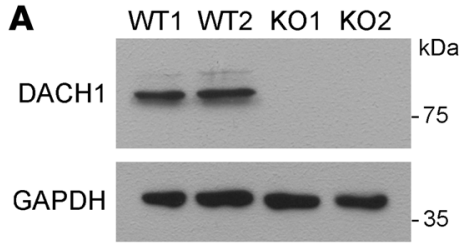

C
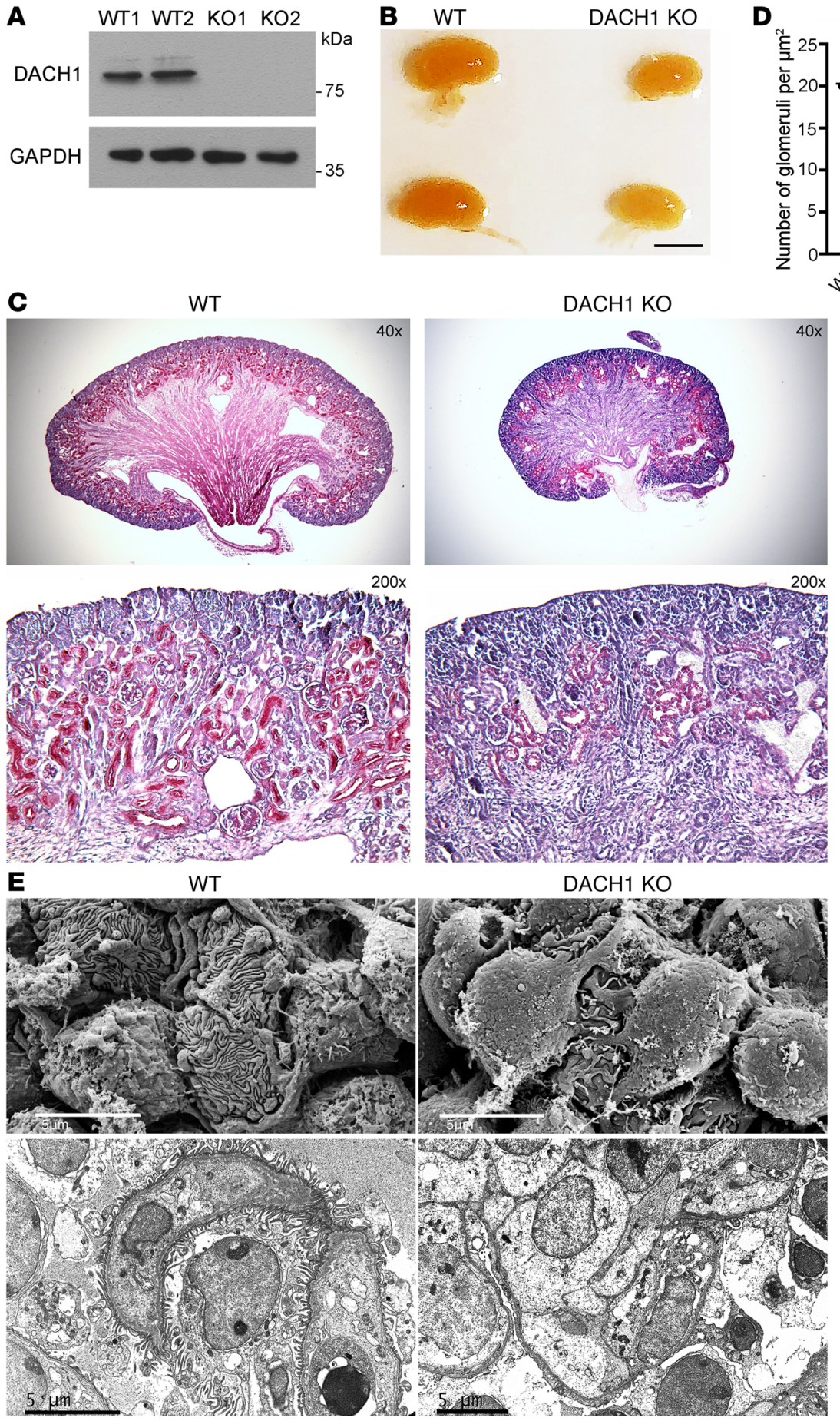

DACH1 KO

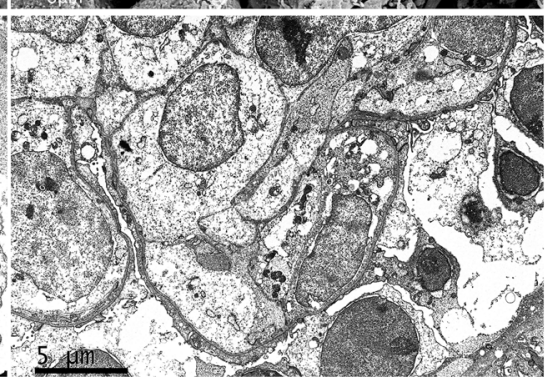

because the strength of its correlation to poor clinical outcomes was greater than for almost any other podocyte protein.

Global Dach1 KO mice demonstrate severe renal hypoplasia and die soon after birth. We generated both Dach1 global and podocyte-specific KO mice using "knockout first" technology from the International Mouse Phenotyping Consortium (IMPC) (29). Similarly to a previously described Dach1 global KO mouse model (1), our Dach1 KO mice survived to birth and appeared grossly similar to WT littermates on the first day of life, but rapidly died prior to the second postnatal day. Western blotting of pooled

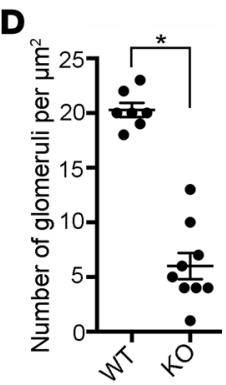

\begin{abstract}
Figure 3. Global Dach1 KO mice manifest renal hypoplasia, podocyte developmental failure, and die perinatally. (A) Western blotting using a DACH1-specific antibody on pooled brain and kidney lysates from WT and Dach1 KO newborn pups. (B) Comparison of kidney gross appearance of newborn WT and global Dach1 KO littermates. Scale bar: $600 \mu \mathrm{m}$. (C) PAS stain of coronal sections from these kidneys shows reduced diameters of nephrogenic and medullary zones with reduced glomerular number. Original magnification, $\times 40$ (upper panels); $\times 200$ (lower panels). (D) Quantification of glomerular density. ${ }^{*} P<0.0001,2$-tailed Student's $t$ test. (E) SEM of newborn WT and Dach1 KO kidneys (upper panels). KO glomeruli show grossly disorganized foot processes that fail to interdigitate. TEM images show severe failure of foot-process formation in newborn Dach1 KO mice compared with WT littermates (lower). Scale bars: $5 \mu \mathrm{m}$.
\end{abstract}

brain and kidney protein lysates collected from newborn mice confirmed absent DACH1 expression in the global KO (Figure 3A). Progeny of heterozygous-heterozygous crossing when genotyped within hours of birth followed expected Mendelian ratios: $22 \mathrm{KO}, 47$ heterozygous, 24 WT. However, by the second postnatal day, no KO mice were detected out of 32 total pups genotyped. The cause of death for the previously reported Dach1 KO model was not determined, and all organs were reported to be morphologically normal (1). Our global Dach1 KO mice, on the other hand, demonstrated severe renal hypoplasia (Figure 3B) without gross abnormalities of other major organs. Similarly, in humans, a patient with congenital renal dysplasia who progressed to ESRD in early childhood and showed no extrarenal manifestations was found to carry a homozygous DACH1 missense mutation (30). Coronal sections of Dach1 global KO kidneys collected on the first day of life showed reduced diameters of medullary and nephrogenic zones (Figure 3C). The number of developing glomeruli within the nephrogenic zone was also dramatically reduced (Figure 3C and quantified in Figure 3D). Scanning electron microscopy (SEM) accentuated the overall limited and disorganized nature of foot-process formation (Figure 3E). Transmission electron microscopic (TEM) analysis of these glomeruli confirmed overall massive podocyte maturation failure with features that included retained columnar appearance with failure to progress to capillary loop stage, mixture of more and less developed appearing podocytes within a single glomerulus, irregularity 
A
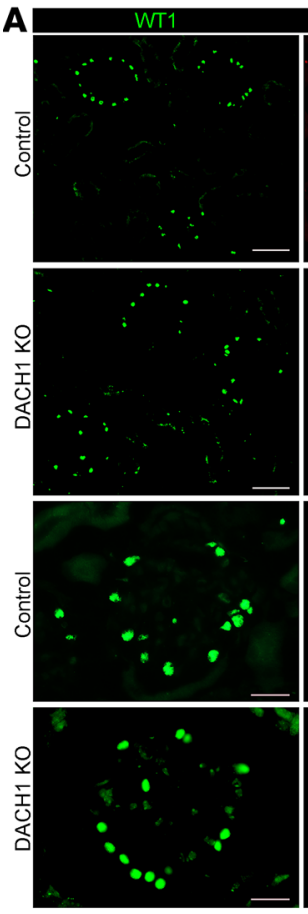

B
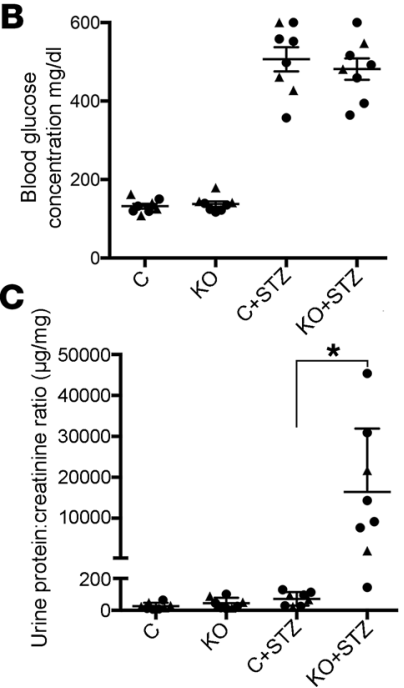
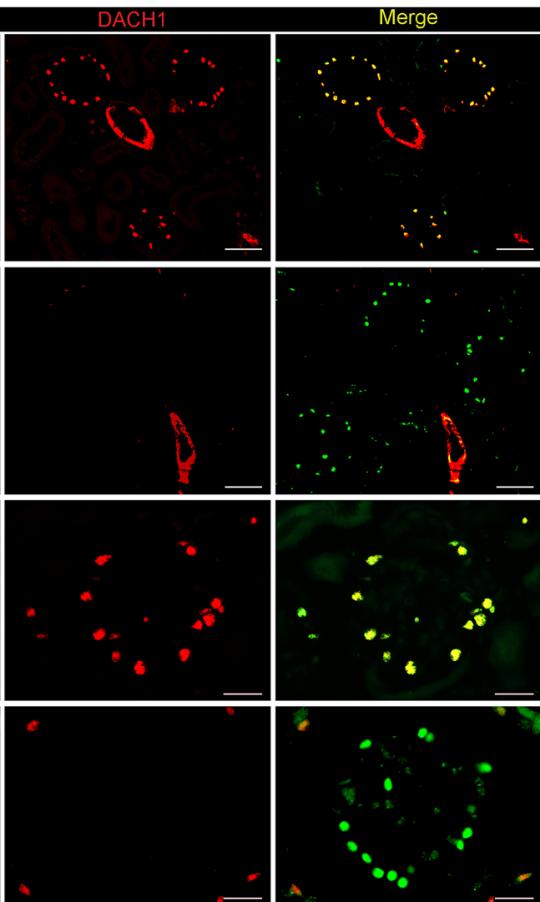

E

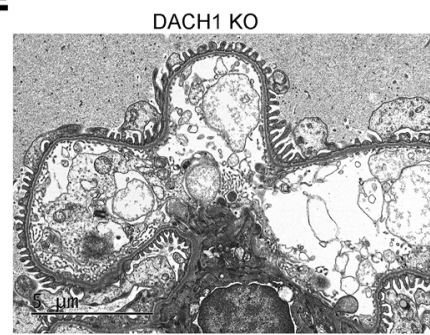

$\mathrm{DACH} 1 \mathrm{KO}+\mathrm{STZ}$ (FSGS)

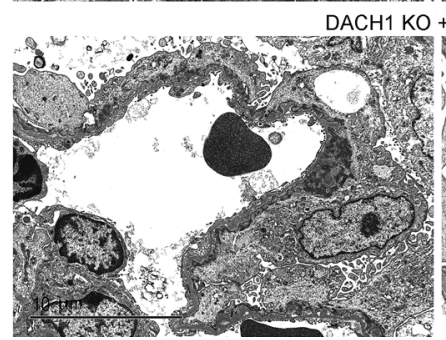

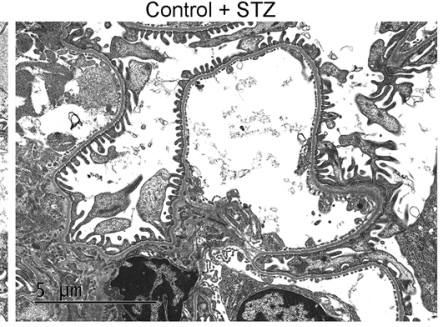
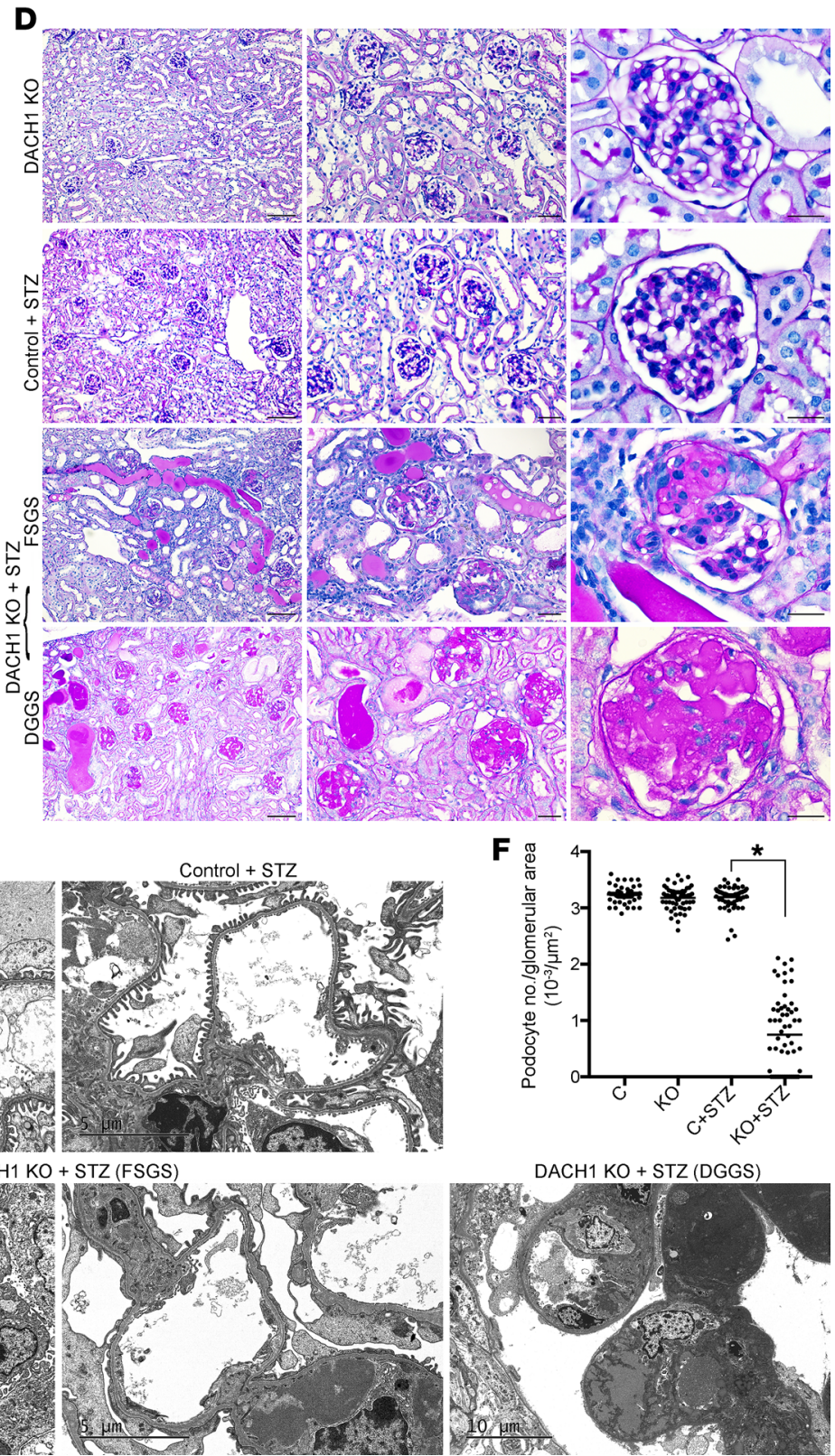

Figure 4. Podocyte-specific Dach1 KO mice, after onset of type I DM, exhibit severe podocyte injury with rapid progression to ESRD. (A) Double-immunofluorescent staining for DACH1 and the podocyte nuclear marker WT1. Podocyte-specific Dach1 KO mice show absent podocyte DACH1 expression, whereas control littermates exhibit robust podocyte DACH1 expression. Scale bars: $50 \mu \mathrm{m}$ (low power); $20 \mu \mathrm{m}$ (high power). (B) Blood glucose levels of mice 2 weeks after completion of STZ administration. Circles, male mice; triangles, female mice. (C) Spot urine protein:creatinine ratios of mice collected 4 weeks after onset of type I DM. * $P$ < 0.01. (D) PAS-stained kidney sections. Nondiabetic age-matched podocyte-specific Dach1 KO mice have normal appearing glomerular morphology (top row). Agematched control mice also demonstrate normal glomerular structure 1 month after onset of type I DM (second row). Podocyte-specific Dach1 KO mice, however, also sacrificed 1 month after DM onset, showed severe FSCS with evidence of podocyte loss and detachment and diffuse tubular proteinaceous casts (third row). In fact, several mice by this time point had progressed to DGCS consistent with ESRD (bottom row). Scale bars: $100 \mu \mathrm{m}$ (low power); $40 \mu \mathrm{m}$ (medium power); 20 $\mu \mathrm{m}$ (high power). (E) Podocyte-specific Dach1 KO mice under basal conditions demonstrate open capillary loops with delicate foot processes (upper left). Control mice also show normal podocyte morphology 1 month after onset of type I DM (upper right). Diabetic podocyte-specific Dach1 KO mice, however, demonstrate catastrophic podocyte injury characterized by total disruption of actin cytoskeletal structure (lower left) with loss of primary and secondary processes and complete foot-process effacement (lower middle). Several mice showed DGCS with severe podocyte loss and a largely denuded GBM (lower right). (F) Quantification of podocyte numbers. $n=3$ mice per group with 20 glomeruli analyzed per mouse. ${ }^{*} P<0.0001$, 2-tailed Student's $t$ test.

of nuclear shape, and overall absence of foot-process formation (Figure 3E). Based on these findings, we suspect that global Dach1 KO mice die from renal failure, although no direct measurements of kidney function were performed.
Podocyte-specific Dach1 KO mice maintain normal glomerular architecture under basal conditions, but develop severe podocyte inju$r y$ with rapid progression to ESRD after onset of diabetes. To investigate how reduced podocyte DACH1 expression levels might affect 
A

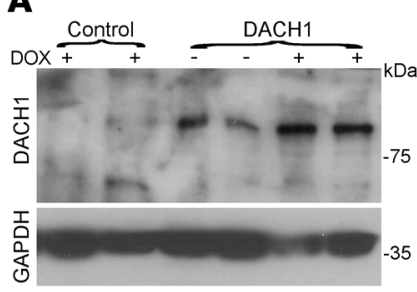

B
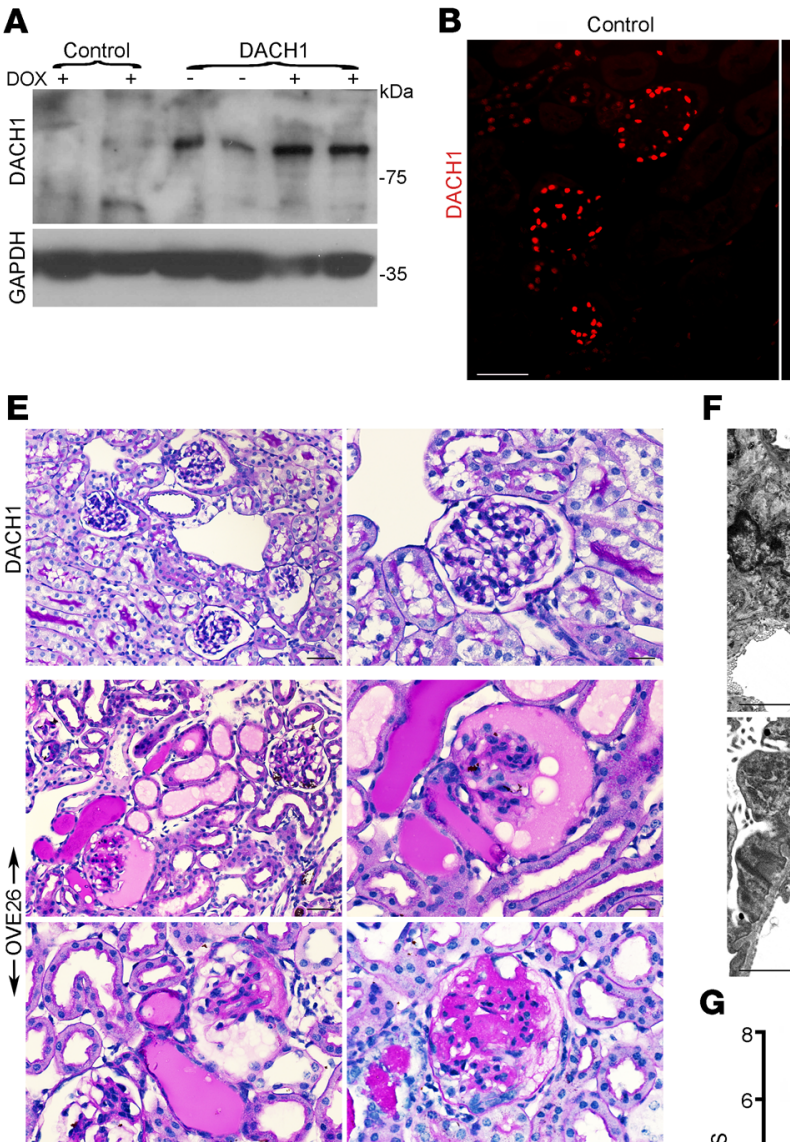

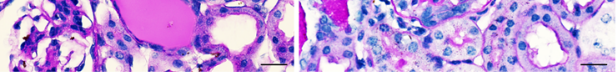

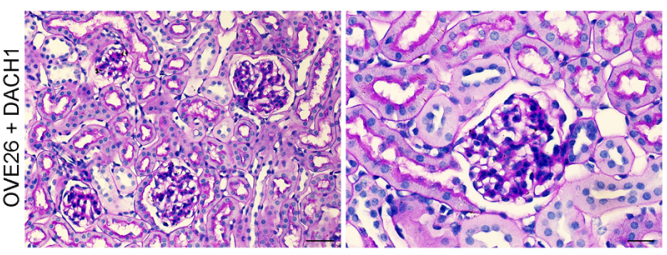

J

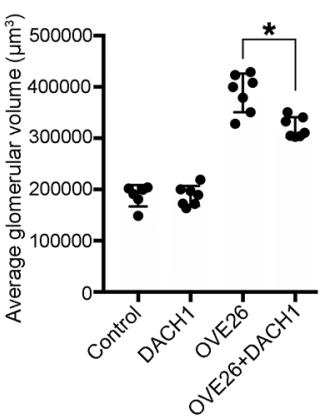

K

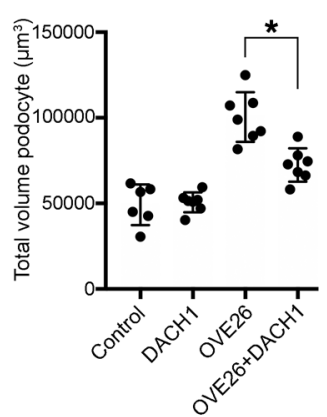

$F$

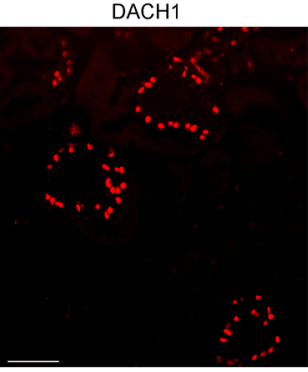

OVE26

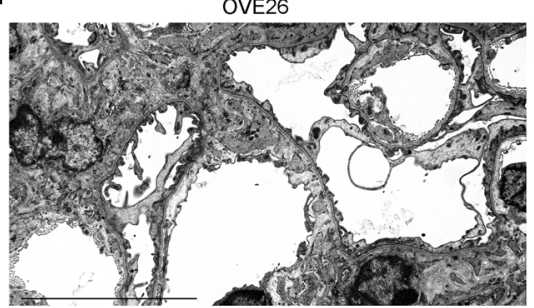

C

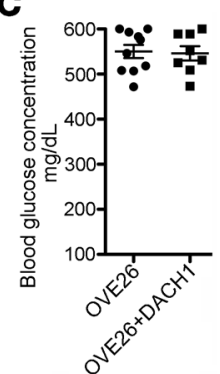

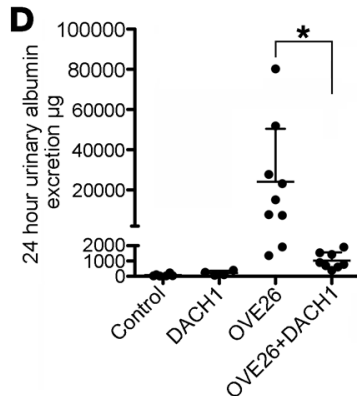

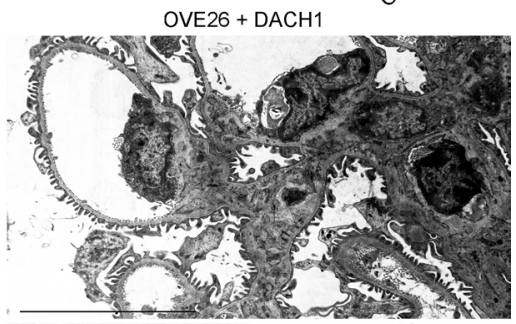

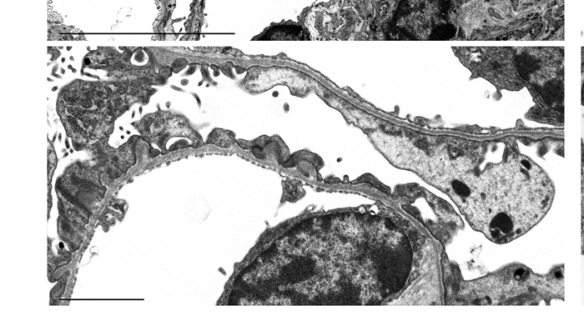

G

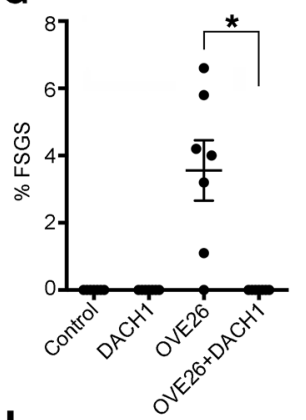

$\mathbf{L}$

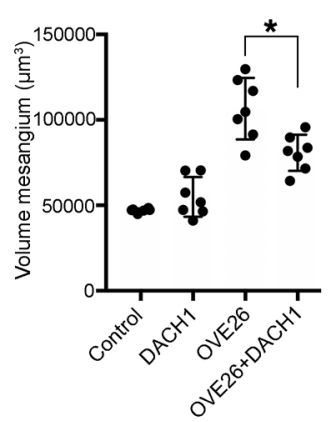

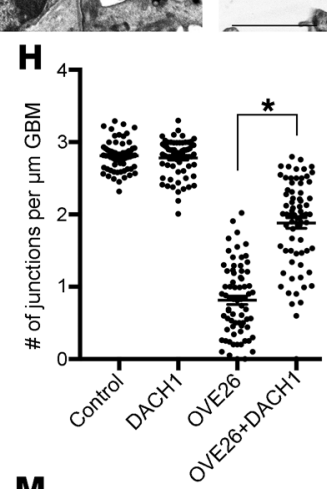

M

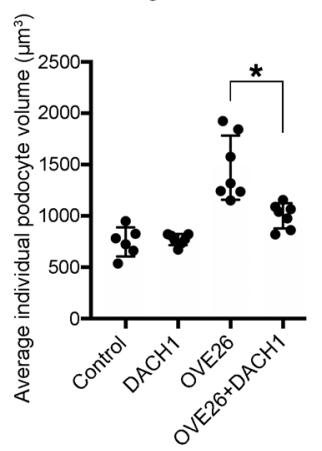

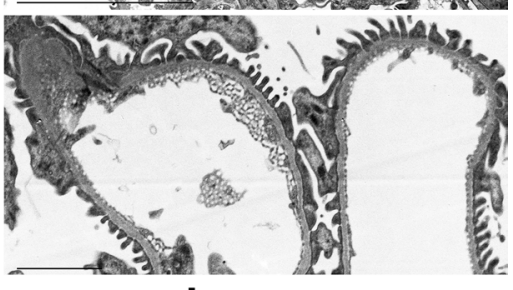

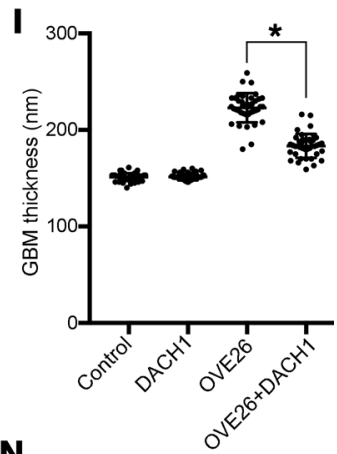

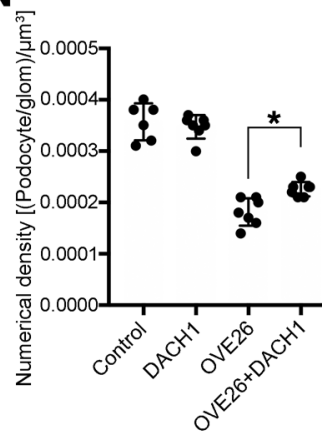

Figure 5. Inducible podocyte-specific DACH1 overexpression protects from DKD. (A) Western blotting using a DACH1-specific antibody on glomerular lysates of transgenic mice. DACH1 mice (TRE-Dach1; pod-rtTA) show robust induction of DACH1 expression after 1 week of DOX supplementation. (B) IF demonstrates that DACH1 overexpression is restricted to podocytes in DACH1 mice. Scale bars: $50 \mu \mathrm{m}$. (C) Blood glucose levels of 6-week-old OVE26 transgenic mice without or with induction of podocyte-specific DACH1 expression. (D) Measurements of 24-hour urinary albumin excretion immediately prior to euthanasia at age of 14 weeks. ${ }^{*} P<0.03$. (E) Representative PAS-stained kidney sections. DACH1 kidneys under basal conditions appear morphologically normal. OVE26 mice show FSCS with associated tubular proteinaceous casts and mesangial expansion. FSGS was not evident in OVE26 littermates that also had podocyte-specific induction of DACH1. Scale bars: $40 \mu \mathrm{m}$ (low power); $20 \mu \mathrm{m}$ (high power). (F) Representative TEM images of OVE26 mice. OVE26 mice demonstrate widespread podocyte injury with loss of primary and secondary processes and diffuse foot-process effacement. OVE26 littermates that also have podocyte-specific expression of DACH1 show significant podocyte protection, including preservation of overall cellular morphology and foot-process architecture. Scale bars: 5 $\mu \mathrm{m}$. (C) Percentage of glomeruli showing FSCS was calculated. ${ }^{*} P<0.002$. (H-N) Morphometric measurements demonstrate that podocyte DACH1 overexpression mitigates the glomerular changes induced by the diabetes of OVE26. Quantification of (H) podocyte effacement $\left({ }^{*} P<0.0001\right)$, (I) CBM thickness $\left({ }^{*} P<0.0001\right)$, (J) average glomerular volume $\left({ }^{*} P<0.0013\right)$, (K) total podocyte volume $\left({ }^{*} P<0.0011\right)$, (L) mesangial volume $\left({ }^{*} P<0.0067\right)$, (M) average individual podocyte volume $\left({ }^{*} P<0.0031\right)$, and $(\mathbf{N})$ podocyte numerical density $\left({ }^{*} P<0.0022\right)$. ( $\mathbf{H}$ and $\left.\mathbf{I}\right) n=3$ mice per group. (J-N) Control, $n=6$ mice; DACH1, OVE26, and OVE26 + DACH1 groups, $n=7$ mice per group. Two-tailed Student's $t$ test (C, D, and G-N). 
A
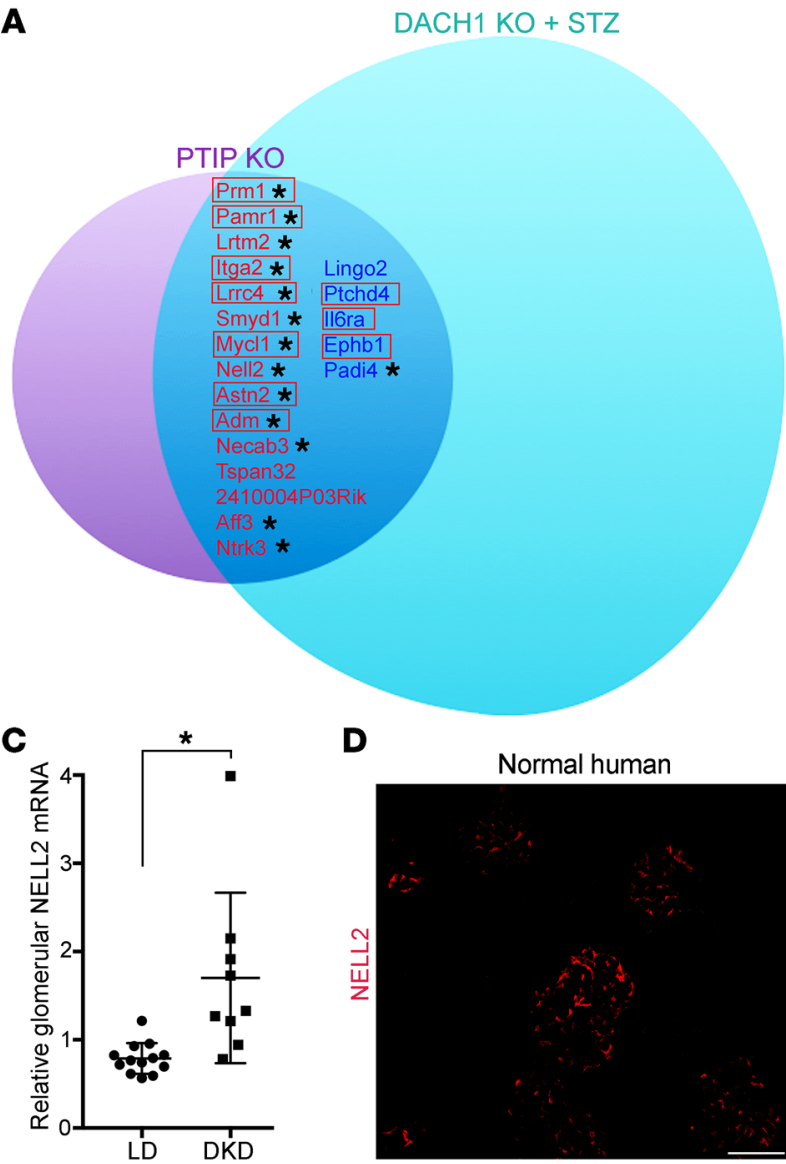

D

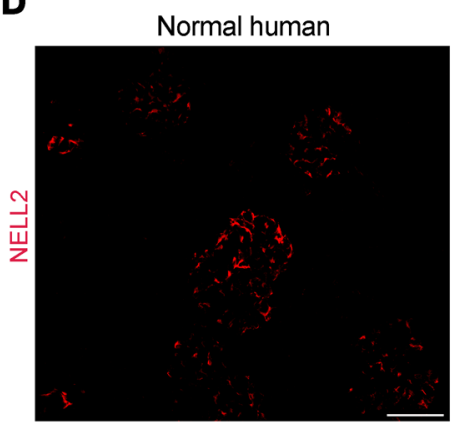

B

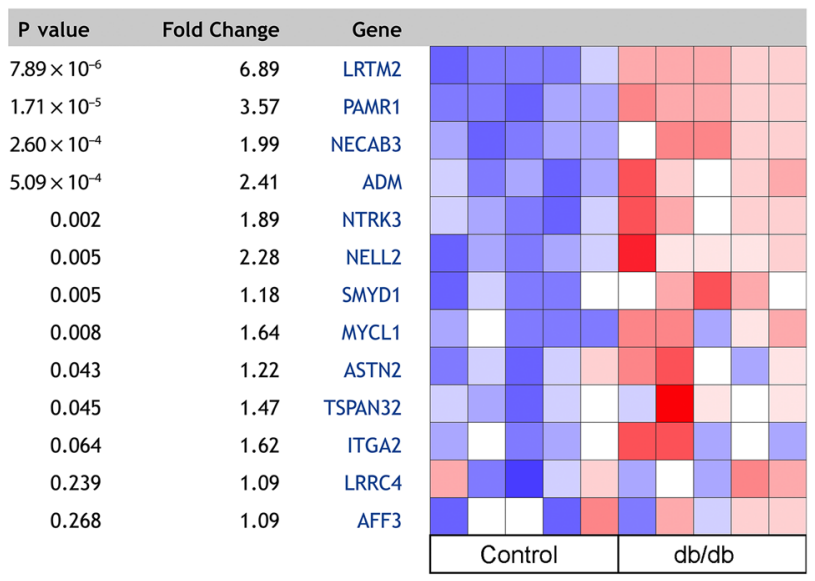

Figure 6. Combining transcriptomic and in silico promoter analyses to identify direct DACH1 transcriptional target genes in podocytes. (A) RNA-Seq was performed to compare glomerular transcriptomes of control and Dach1 podocyte-specific KO mice at baseline and early after STZ-induced DM. Results were overlapped with a previously reported microarray of podocyte-specific Ptip KO mice under basal conditions (38). Twenty out of a total of 38 genes from the Ptip KO glomeruli were also dysregulated in Dach1 KO glomeruli. Genome-wide in silico promoter analysis using a previously reported positional weight matrix (4) found the upregulated genes in this Dach1-Ptip overlap set to be highly enriched for the presence of at least 1 promoter DBD. Cene names surrounded by a red rectangle indicate dysregulation in glomeruli of Dach1 KO mice under basal conditions. Asterisks indicate the presence of promoter DBD. (B) Glomerular mRNA levels of the upregulated Dach1-Ptip overlap gene set in a previously reported microarray analysis of $d b / d b$ mice (39) and subjected to further analysis by Nephroseq. All genes present on the microarray chip from the overlap set are also upregulated to varying degrees in this diabetes model. (C) Glomerular mRNA levels of the DACH1 target gene NELL2 are increased in human DKD. Data are from a previously reported microarray study (40) and subjected to further analysis by Nephroseq. ${ }^{*} P<0.001$. (D) NELL2 fluorescent staining intensity is increased in glomeruli in human nephrectomy samples with a clinicopathological diagnosis of DKD $(n=3)$ compared with normal controls $(n=4)$. Representative images taken with identical exposure times are shown. In kidney overall, NELL2 expression is highly enriched in podocytes. Scale bars: $100 \mu \mathrm{m}$. (E) Differences in NELL2 glomerular fluorescent intensity between DKD ( $n=50)$ and control $(n=50)$ were quantified relative to DAPI $\left({ }^{*} P<0.0005\right)$ and to WT1 $\left({ }^{* *} P<0.0001\right)$. (C and E) Two-tailed Student's $t$ test.

DKD pathogenesis, we analyzed podocyte-specific Dach1 KO mice. Double-immunofluorescent studies demonstrated nearly absent podocyte DACH1 expression, but preserved Wilms tumor 1 (WT1) staining, in podocyte-specific Dach1 KO mice (Figure 4A). Control littermates showed robust podocyte nuclear DACH1 staining that colocalized with WT1. Under basal conditions, podocyte-specific Dach1 KO mice did not manifest proteinuria (Figure 4C), maintained normal glomerular structure into adulthood (Figure 4D), and had normal appearing foot-process architecture on electron microscopy (Figure 4E). To test the susceptibility of these mice to the diabetic condition, we utilized the low-dose streptozotosin (STZ) model, which mimics type I diabetes mellitus (DM) and is typically an early model of DKD with only mild renal histopathological changes even after many months of diabetes. After STZ treatment, both control and Dach1 KO mice developed identical levels of hyperglycemia (Figure 4B). Surprisingly, hyperglycemic podocyte-specific Dach1 KO mice rapidly manifested severe proteinuria (Figure 4C) and many appeared moribund by 1 month after diabetes onset. At this point, mice were euthanized and kidneys harvested. KO kidneys showed massive podocyte injury with severe focal segmental glomerulosclerosis (FSGS), and several mice had already progressed to ESRD, characterized by diffuse global glomerulosclerosis (DGGS) (Figure 4D). Observed histological changes in KO mice with FSGS included moderate mesangial expansion, an established general response to severe ongoing podocyte loss $(17,31,32)$. Marked parietal cell activation, another established glomerular response to severe podocyte loss (33), was also evident in KO glomeruli, as determined by Ki-67 staining (Supplemental Figure 2). Control STZ diabetic mice, as expected, showed no proteinuria and no overt renal histological changes after an identical dura- 
A

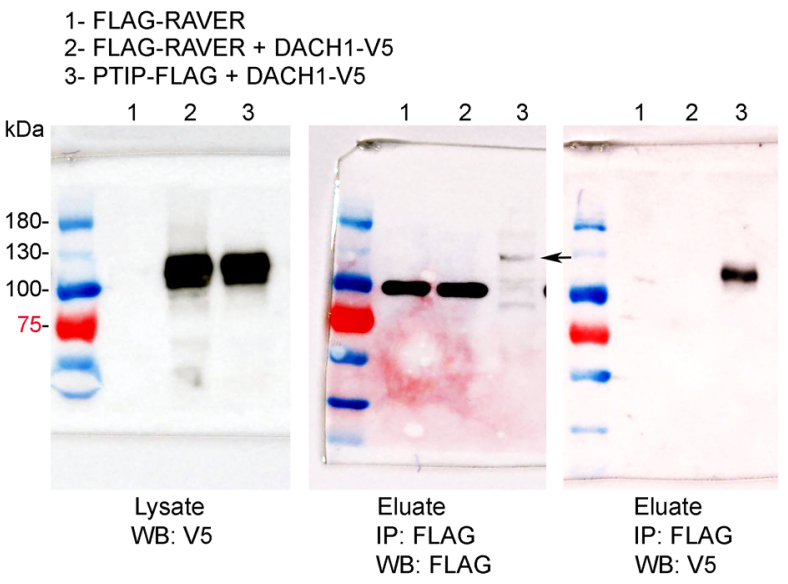

C

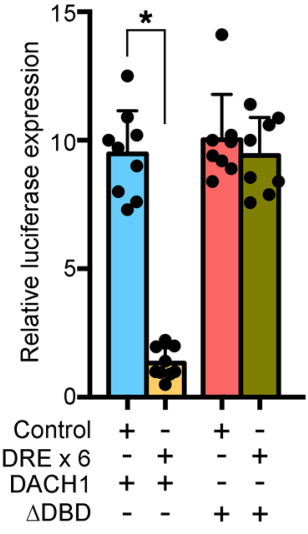

B

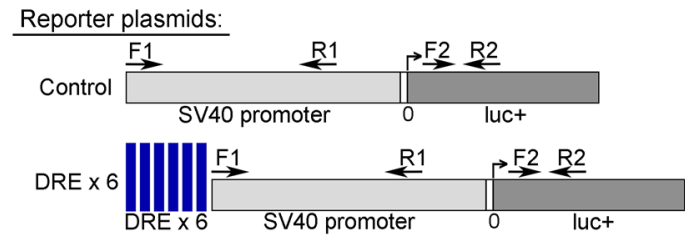

$\underline{\mathrm{DACH} 1 \text { expression plasmids: }}$
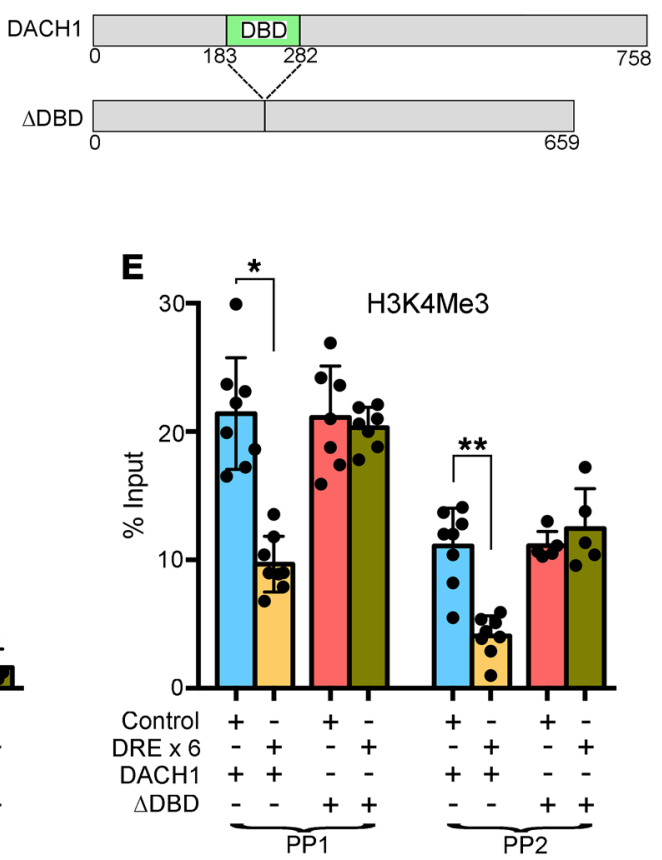

Figure 7. PTIP is recruited by DACH1 to DACH1 DNA binding sites and causes transcriptional repression and decreased H3K4Me3 levels. (A) Co-IP was performed between PTIP-FLAG and DACH1-V5 using antibodies to their tags. FLAG-RAVER was used as a negative control protein. Indicated lysates were combined and incubated overnight with anti-FLAG antibodies bound to superparamagnetic iron-impregnated beads. A robust protein complex is present between PTIP-FLAG and DACH1-V5, but completely absent between FLAG-RAVER and DACH1-V5. Molecular weight of indicated proteins is as follows: FLAG-RAVER, 95 kDa; DACH1-V5, 105 kDa; PTIP-FLAG, 120 kDa. Protein interaction is robust despite comparatively low expression of PTIP-FLAG. Band indicated by arrow. (B) Schematic of SV40-luciferase reporter plasmids (upper) and DACH1 expression plasmids (lower). DREs were synthesized as a sextet multimer and subcloned immediately upstream of the SV40 promoter. Location of primers used for ChIP-qPCR are indicated. The DACH1 $\triangle D B D$ expression plasmid carries a deletion of its box-N domain, which mediates direct binding of DACH1 to DNA. (C) Each of the reporter plasmids was cotransfected with a DACH1 expression plasmid into 293T cells and then luciferase expression measured. Dramatic transcriptional repression was induced by the combined presence of upstream DACH1 DNA binding sites and a DACH1 protein able to bind to these sites. ${ }^{*} P<0.0001$. (D) ChIP-qPCR was performed using an antibody specific for PTIP and primer pairs indicated in B. ${ }^{*} P<0.0001$; ${ }^{* *} P<0.0001$. (E) ChIP-qPCR as in D, but with an H3K4Me3 antibody. ${ }^{*} P<0.0001 ;{ }^{* *} P<$ 0.0001. (C-E) One-way ANOVA and Tukey's post hoc test.

tion of hyperglycemia. Electron microscopic analysis emphasized the extreme susceptibility of podocyte-specific Dach1 KO mice to the diabetic condition. One month after onset of diabetes, these podocytes demonstrated catastrophic cellular injury characterized by severe disruption of actin cytoskeletal structure, loss of primary and secondary processes with complete effacement (Figure 4E), and progression to global glomerular sclerosis with massive loss of overlying podocytes, leaving the glomerular basement membrane (GBM) completely denuded (Figure 4E). Control mice that were exposed to hyperglycemia of identical duration showed normal appearing podocytes with intricate foot-process formation and no effacement (Figure 4E). To quantify podocyte number, we counted WT1-positive cells per glomerular area. As expected, podocyte loss was severe in podocyte-specific Dach1 KO mice after exposure to hyperglycemia (Figure $4 \mathrm{~F}$ ).
To test the susceptibility of podocyte-specific Dach1 KO mice to a second form of podocyte injury, we analyzed their response to adriamycin (ADR) nephropathy, a podocyte-injury model that is highly dependent on genetic background. These mice developed massive proteinuria within the first week or 2 after ADR injection (Supplemental Figure 3A) with early lethality from renal failure. Pathology revealed severe and diffuse FSGS with widespread tubular proteinaceous casts (Supplemental Figure 3B). Control mice, on the other hand, were completely resistant to development of nephropathy. The severity of ADR-induced podocyte injury was accentuated by electron microscopic analysis (Supplemental Figure 4). Two weeks after ADR injection, podocytes demonstrated catastrophic cellular injury manifested by massive microvillous transformation with severe blebbing and cytoplasmic vacuolization, complete loss 
A
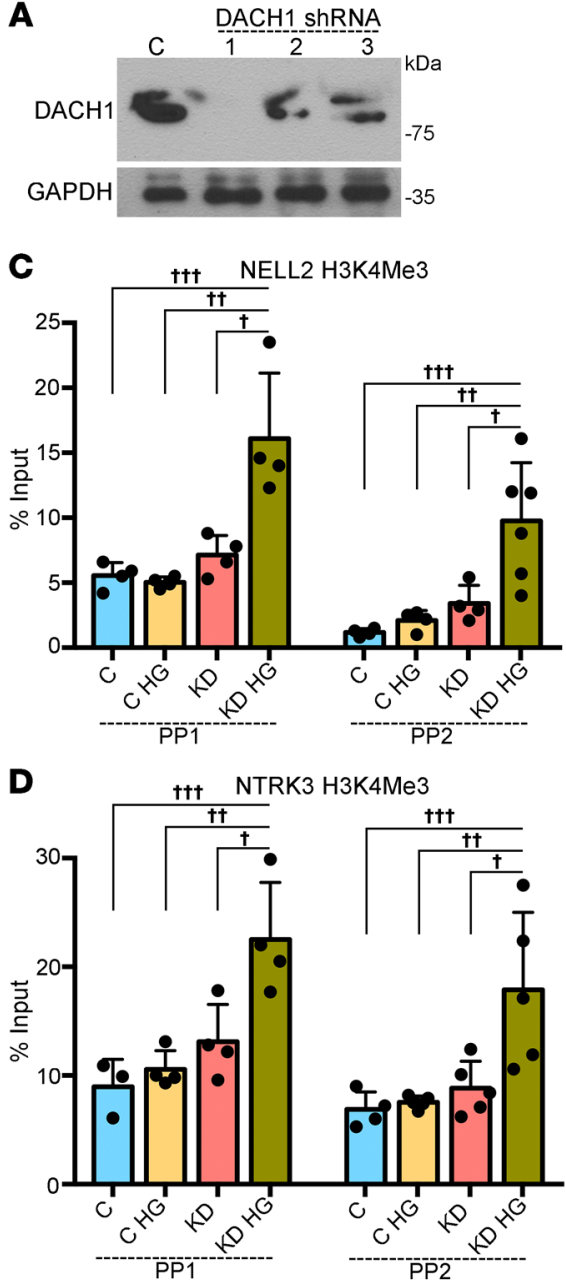

B
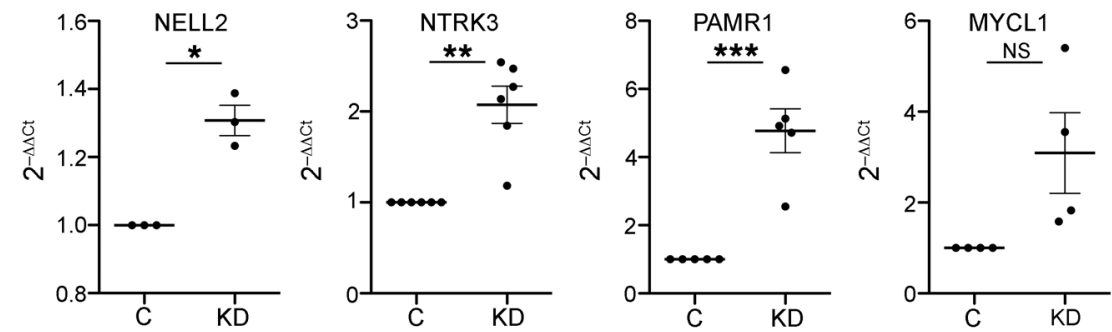

Control, mannitol vs. hyperglycemia: $\longrightarrow$
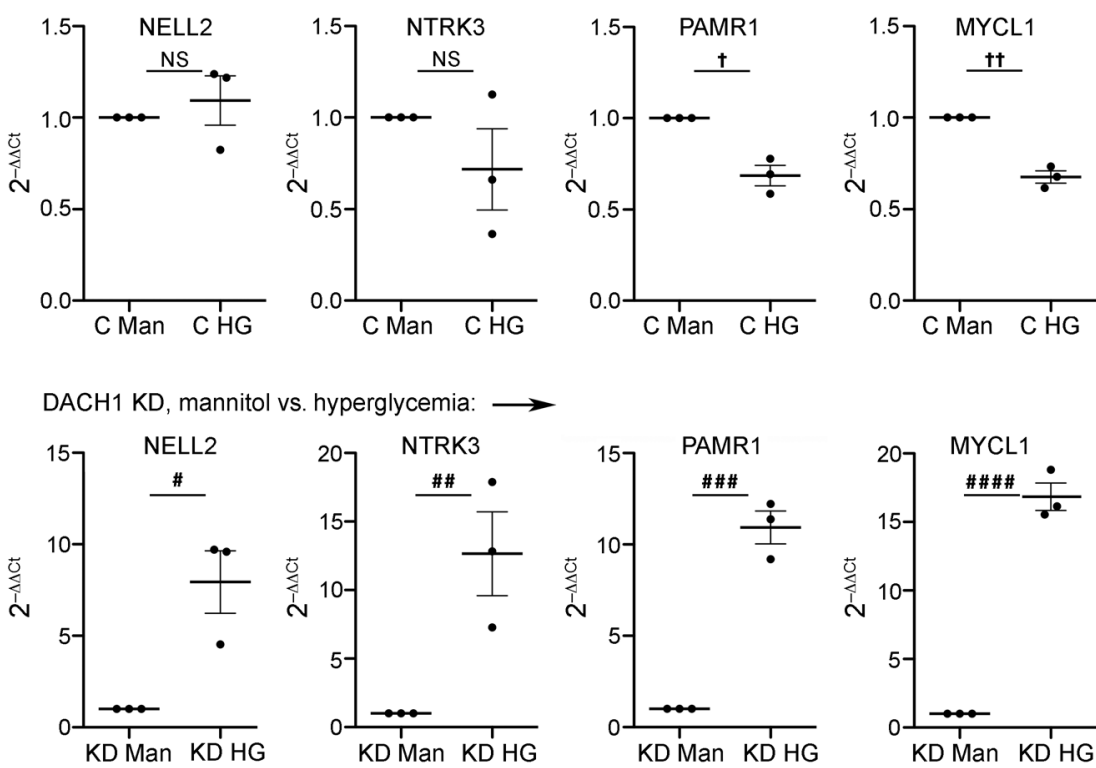

Figure 8. DACH1 knockdown in podocytes combined with hyperglycemia triggers target gene derepression and increases promoter H3K4Me3 levels. (A) Western blot comparing levels of DACH1 protein between stably transduced human podocyte cell lines that each express a distinct DACH1-targeted shRNA or scrambled shRNA control plasmid. (B) qPCR for 4 DACH1-target genes (NELL2, NTRK3, PAMR1, and MYCL1) in DACH1 knockdown shRNA no. 1 and control transduced podocytes with and without hyperglycemia. Under basal conditions, DACH1 knockdown (KD) podocytes showed modest transcriptional derepression of each of the 4 genes compared with control shRNA podocytes (top panel). ${ }^{*} P<0.003 ;{ }^{* *} P<0.0004 ;{ }^{* * *} P<0.0004$. In control podocytes cultured in high glucose, none of the 4 genes demonstrated increased mRNA expression levels compared with identical cells grown in mannitol (middle panel). ${ }^{\dagger} P<$ 0.005 ; ${ }^{+\dagger} P<0.0007$. In DACH1 KD podocytes grown in high glucose, however, transcriptional derepression was augmented dramatically (lower panel). ${ }^{\#} P<$

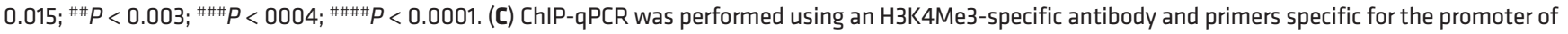
NELL2 comparing chromatin extracted from DACH1 KD and control podocytes with and without high glucose. The combination of DACH1 KD with hyperglycemia caused an increase in levels of promoter bound H3K4Me3. Primer pair 1: ${ }^{*} P<0.002 ;{ }^{* *} P<0.001 ;{ }^{* *} P<0.001$. Primer pair $2:{ }^{\dagger} P<0.015 ;{ }^{+t} P<0.004 ;{ }^{+t+} P<$ 0.001. (D) ChIP qPCR as in C, but with primers specific for the NTRK3 promoter. Primer pair $1:{ }^{*} P<0.015 ;{ }^{* *} P<0.003 ;{ }^{* * *} P<0.002$. Primer pair 2 : ${ }^{\dagger} P<0.012$; ${ }^{t+} P<0.004$; ${ }^{t+t} P<0.004$. (B) Two-tailed Student's $t$ test. (C and $\left.\mathbf{D}\right)$ One-way ANOVA and Tukey's post hoc test.

of primary and secondary processes with total foot-process effacement, and large areas of podocyte detachment. Podocytes in ADR-treated control mice, on the other hand, showed only mild patchy areas of foot-process effacement with preservation of overall cellular morphology.

Preventing diabetes-induced loss of podocyte DACH1 expression slows DKD progression. Human DKD patients have diminished podocyte DACH1 expression levels, and loss of podocyte DACH1 in mice dramatically enhances susceptibility to the hyperglycemic condition. However, whether augmenting levels of podocyte DACH1 alone is sufficient to reduce podocyte injury and mitigate DKD progression is not known. To investigate this, we generated transgenic mice with inducible podocyte-specific expression of
DACH1 using the "tet-on" system. In this system, gene activation is driven from the Tet-responsive element (TRE) only after activation by the rtTA regulatory protein and in the presence of doxycycline (DOX). Transgenic mice that express rtTA specifically in podocytes (podocin-rtTA) were bred to TRE-Dach1 transgenic mice. After 10 days of DOX administration, double-transgenic mice (podocin-rtTA; TRE-Dach1) showed robust induction of glomerular DACH1 by Western blotting (Figure 5A). Based on IF using a DACH1-specific antibody, DACH1 induction was limited to podocytes only, with fainter areas of tubular expression appearing as identical between control and Dach1 transgenic kidneys (Figure 5B). However, double-transgenic mice also showed modestly increased glomerular DACH1 expression levels even without 
A

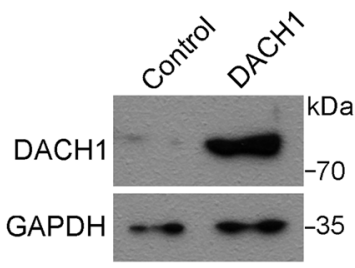

B NELL2 promoter:

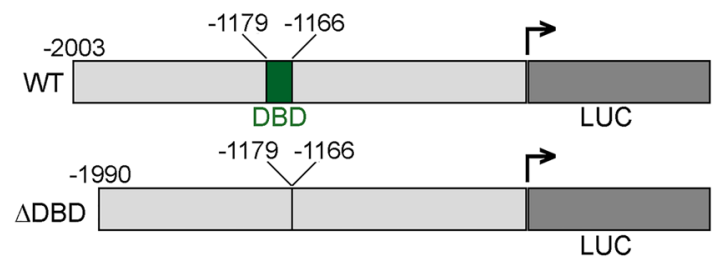

C

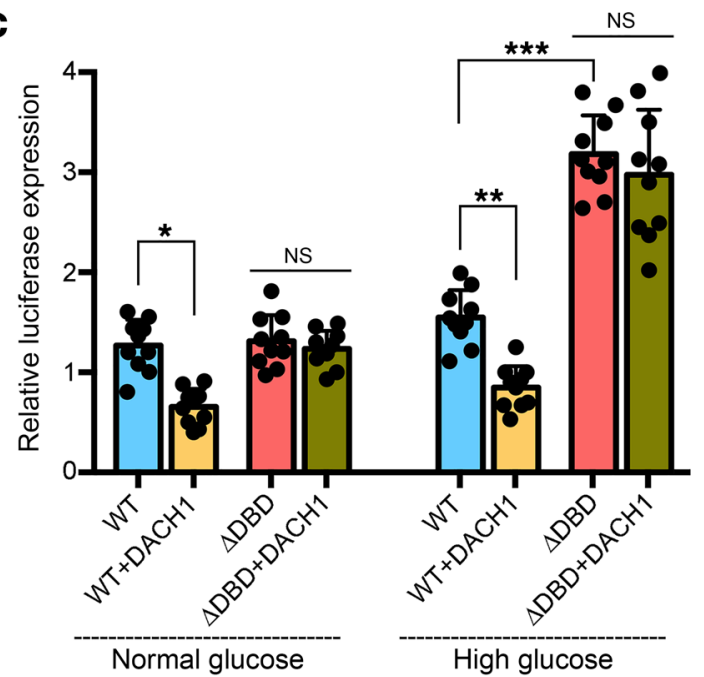

DOX administration, suggesting promoter leakage (Figure 5A). To account for this, we did not perform any experiments that compare mice with and without DOX administration. These double-transgenic mice were bred to OVE26 diabetic mice, a transgenic mouse line that spontaneously develops severe type I diabetes on the first day of life, to generate triple-transgenic mice (podocin-rtTA; TREDach1; OVE26) that were compared with double-transgenic mice (podocin-rtTA; OVE26). All mice were fed DOX chow from weaning age until time of euthanasia.

Despite equal levels of hyperglycemia (Figure 5C), OVE26 mice with podocyte-specific induction of DACH1 showed significantly reduced proteinuria compared with OVE26 control mice, as determined by a 24-hour urine collection immediately prior to euthanasia at 14 weeks of age (Figure 5D). OVE26 mice are characterized by severe podocyte injury with FSGS (34). Podocyte-specific induction of DACH1 completely abrogated the OVE26 FSGS phenotype compared with control OVE26 mice (Figure 5E and quantified in Figure 5G). Control OVE26 mice showed FSGS with associated areas of diffuse tubular proteinaceous cast formation. We could not detect even a single FSGS lesion in any of the age-matched OVE26 mice that also had podocyte-specific induction of DACH1. TEM showed striking preservation of foot-process architecture and normalization of podo-
Figure 9. DACH1-mediated transcriptional repression in podocytes requires direct DACH1 sequence-specific promoter binding. (A) Western blot to compare levels of DACH1 protein between human podocytes stably transduced with a DACH1 expression plasmid and podocytes stably transduced with vector alone. (B) Schematic of luciferase lentiviral reporter constructs. Shown are the full-length NELL2 promoter (WT) and one that contains a deletion of its DBD ( $\triangle \mathrm{DBD}$ ). (C) In WT transduced podocytes, NELL2 promoter activity was lower in DACH1 overexpressing podocytes compared with controls both at baseline $\left({ }^{*} P<0.0001\right)$ and under conditions of hyperglycemia ( $\left.{ }^{* *} P<0.0001\right)$. In $\triangle \mathrm{DBD}$ transduced podocytes, however, DACH1-mediated transcriptional repression was absent, and luciferase levels were not different between DACH1 overexpressing and control cells both at baseline and in hyperglycemia. Moreover, when exposed to hyperglycemia, $\triangle \mathrm{DBD}$ promoter activity was significantly higher than that of the WT promoter $\left({ }^{* *} P<0.0001\right)$, a pattern similar to transcriptional derepression evident in DACH1 knockdown podocytes. (C) One-way ANOVA and Tukey's post hoc test.

cyte cellular shape in OVE26 mice that had podocyte-specific DACH1 induction compared with control OVE26 mice, which demonstrated diffuse foot-process effacement, loss of primary and secondary processes, and abnormal cellular morphology (Figure 5F). In a blinded fashion, we performed detailed glomerular morphometric analysis of kidneys from the 4 groups of mice: control, podocyte-specific DACH1 overexpression, OVE26, and OVE26 with podocyte DACH1 overexpression. Levels of podocyte effacement (Figure 5H) and GBM thickening (Figure 5I) were markedly lessened in OVE26 mice that also had podocyte DACH1 overexpression. In OVE26 mice, similarly to what occurs in human DKD, overall glomerular size, total podocyte volume, individual podocyte volume, and mesangial volume were each drastically increased, and podocyte density was decreased (Figure $5, \mathrm{~J}-\mathrm{N}$ ). Each of these changes was substantially mitigated by concomitant podocyte DACH1 overexpression. Importantly, the morphometric analysis did not identify any significant differences between control and podocyte-specific DACH1 overexpressing mice. This is consistent with our observations of absent proteinuria and normal light and electron microscopy in these mice. Overall, these findings suggest that podocyte DACH1 strongly protects from diabetic glomerular injury induced in OVE26 mice.

Identification of direct DACH1 transcriptional target genes in podocytes. To identify direct transcriptional targets of DACH1 in podocytes, we combined glomerular transcriptomic studies of podocyte-specific Dach1 KO mice with in silico promoter analysis. RNA-Seq was performed to compare glomerular transcriptomes of control and Dach1 podocyte-specific KO mice early after STZ-induced diabetes (Figure 6A). An early time point was intentionally selected to better focus on direct DACH1 target genes, a goal that would become more difficult once podocyte injury had become severe. RNA-Seq was also performed to compare podocyte-specific Dach1 KO with control mice under the basal conditions in which podocyte morphology is normal, but susceptibility to injury extremely high. Transcriptional levels of major podocyte differentiation markers (such as Nphs1, Nphs2, or Magi2) showed no significant differences between groups, suggesting that DACH1 is not directly involved in driving transcription of major podocyte differentiation markers. Surprisingly, the most upregulated glomerular gene in Dach1 KO mice, both at baseline (560-fold upregulation) and in early DM (2325-fold increase), was 


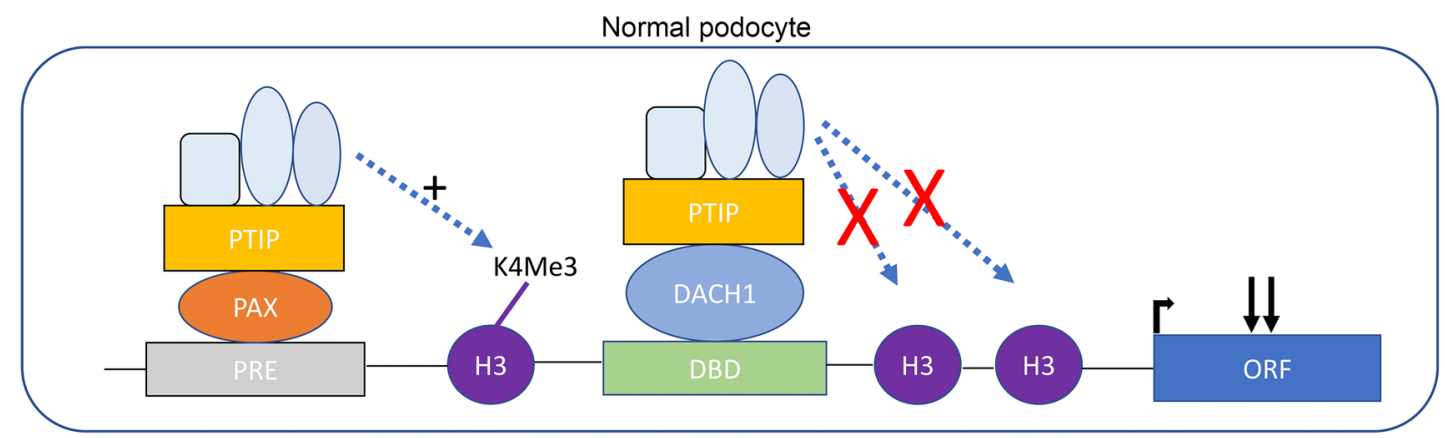

Diabetic kidney disease podocyte

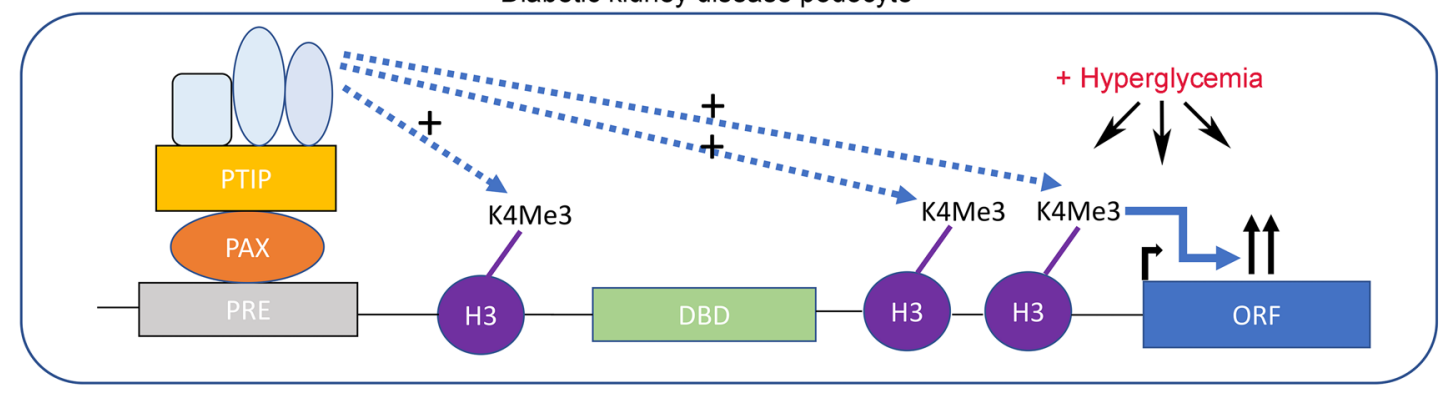

Figure 10. Proposed mechanism of transcriptional derepression of DACH1 target genes in human DKD. Previous studies show that PTIP is recruited by Pax proteins to promoter Pax response elements (PREs) and that this enhances promoter H3K4Me3 levels and causes transcriptional activation (24). In the current work, we show that PTIP can also be recruited by sequence-specific binding of DACH1 to its target gene promoters, but that this recruitment suppresses transcription and reduces promoter H3K4Me3 levels. We propose that in DKD, reduced levels of podocyte DACH1 expression cause diminished recruitment of PTIP by DACH1 to its target gene promoters. This, in combination with hyperglycemia, results in increased promoter H3K4Me3 levels and transcriptional derepression of multiple DACH1 target genes.

protamine-1 (Prm1), which is highly expressed in spermatozoa, is essential for their maturation, and whose expression is highly regulated by histone methylation (35-37). Comprehensive literature and database searches of all previously published glomerular transcriptomic data sets revealed only one other where Prm1 mRNA expression was significantly dysregulated: a microarray analysis of glomeruli from podocyte-specific Ptip KO mice under basal conditions (38). Further comparison of the podocyte-specific Ptip and podocyte-specific Dach1 KO transcriptomic data sets revealed that of 38 total dysregulated genes for Ptip KO glomeruli, 20 were also dysregulated for Dach1 KO glomeruli early after diabetes onset (Figure 6A and Supplemental Figure 5). Importantly, the majority of overlapping genes were dysregulated in opposing directions: downregulated in Ptip KO and upregulated in Dach1 KO.

Next, we performed in silico promoter analysis of the 20 genes in the overlap set to look for direct Dach1-binding sites in their promoters (Figure 6A). TRANSFAC promoter analysis was performed using a previously reported DACH1 positional weight matrix (4) in the region of -2500 to +500 of the ATG start site. We allowed for either exact or single-nucleotide mismatches, as was reported previously (4). Of the 15 genes in the overlap set that were upregulated in Dach1 KO glomeruli, 13 contained at least one Dach1 binding domain (DBD) in the promoter $(13 / 15,86.7 \%)$ compared with an overall genome-wide rate of $18.2 \%$. For these 15 genes, promoter sequence data derived from the National Center for Biotechnology Information (NCBI) were manually reviewed to confirm the presence and location of DBDs. Enrichment for promoter DBDs was not evident for the 5 genes downregulated in Dach1 KO glom- eruli that overlapped with Ptip KO glomeruli, where only 1 of $5(1 / 5,20 \%)$ contained at least 1 promoter DBD. The high DBD enrichment rates for genes in the upregulated gene set suggested that these genes are likely direct DACH1 targets, that the loss of direct DACH1 promoter DNA binding may lead to transcriptional derepression of these genes, and that the mechanism of derepression may involve PTIP.

In order to validate the upregulated Dach1-Ptip overlap gene set as potentially relevant in DKD pathogenesis, we analyzed mouse and human DKD glomerular transcriptomic data sets available in Nephroseq. We found that all genes from the upregulated Dach1-Ptip overlap set were also upregulated to varying degrees in glomeruli from $d b / d b$ diabetic mice (39) (Figure 6B) and that this upregulation was severe in many cases. In transcriptomic analysis of glomeruli from human DKD patients, the DACH1 target gene NELL2 was significantly upregulated (Figure 6C) (40). We performed quantitative immunofluorescent analysis for NELL2 on frozen kidney sections taken from control and DKD patients who had undergone nephrectomy. We found NELL2 to be specifically expressed in podocytes in kidney and that podocyte NELL2 expression was significantly enhanced in human DKD compared with in controls (Figure 6D, quantified in Figure 6E). Because WT1 expression was reduced in DKD (but DAPI is unchanged), the differences in NELL2 intensity measurements between control and DKD were higher when normalization was performed relative to WT1. This emphasizes that overall podocyte NELL2 expression is increased in spite of diminished podocyte number, suggesting a high level of NELL2 upregulation per individual podocyte. 
Recruitment of PTIP by DACH1 to the promoters of DACH1 target genes causes transcriptional repression and diminished H3K4Me3 levels. To investigate whether PTIP has a role in DACH1-mediated transcriptional repression, we performed co-IP for V5-tagged DACH1 using FLAG-tagged PTIP as bait (Figure 7A). We demonstrate a robust protein/protein interaction between PTIP-FLAG and DACH1-V5, but no interaction between the negative control bait protein FLAG-tagged RAVER and DACH1-V5. Next, to determine whether DNA-bound DACH1 can recruit PTIP to the promoter of DACH1 target genes, we cloned the DACH1 response element (DRE) as a multimer into the pGL3-control luciferase reporter plasmid in a manner similar to what was previously described (5). The resulting plasmid contained 6 DREs in series immediately upstream of the SV40 promoter, which was compared with the control plasmid that contained the SV40 promoter alone (Figure 7B). We also generated a mutant DACH1 expression plasmid that carried a deletion of its box-N domain, which mediates its direct DNA binding $(4,41)$ (Figure 7B). When these plasmids were cotransfected into $293 \mathrm{~T}$ cells, SV40 promoter activity was markedly repressed by the presence of both DACH1 DNA-binding sites and a full-length DACH1 version capable of binding to these sites (Figure 7C). The DACH1 mutant variant that was unable to bind to DNA did not repress transcription. ChIP-quantitative PCR (ChIP-qPCR) was then performed with a PTIP antibody using 2 distinct primer pairs whose locations are shown in Figure 7B. The results revealed a strong increase in promoter-bound PTIP in the setting of DACH1 DNA-mediated transcriptional repression (Figure 7D). The mutant DACH1 variant that was unable to bind DACH1 did not mediate PTIP recruitment to DNA. Our next question was whether the recruitment of PTIP by DACH1 influenced H3K4 methyltransferase complex activity. To answer this, we again performed ChIP-qPCR using the same primer pairs, but substituting an antibody specific for H3K4Me3. We found decreased levels of promoter $\mathrm{H} 3 \mathrm{~K} 4 \mathrm{Me} 3$ in the setting of DACH1-mediated recruitment of PTIP to DNA (Figure 7E). This effect was abrogated when expressing the DACH1 mutant version that cannot bind DNA.

DACH1 knockdown in podocytes combined with hyperglycemia triggers target gene upregulation and increases promoter $\mathrm{H} 3 \mathrm{~K} 4 \mathrm{Me} 3$ levels. Next, we investigated whether transcriptional derepression of DACH1 target genes, as occurs in podocytes in $\mathrm{DKD}$, is caused by increases in promoter $\mathrm{H} 3 \mathrm{~K} 4 \mathrm{Me} 3$ levels. To accomplish this, we generated DACH1 knockdown podocyte cell lines by stably transducing each with a distinct $D A C H 1$-targeted shRNA expression plasmid and compared them with podocytes stably transduced with a scrambled shRNA control plasmid (Figure 8A). By Western blotting, podocytes that expressed DACH1 shRNA no. 1 showed substantially reduced levels of DACH1 protein and were utilized for further studies. To validate that these DACH1 knockdown podocytes recapitulated our prior observations from podocyte-specific Dach1 KO mice, we performed qPCR for 4 genes from the Dach1-Ptip overlap gene set: NELL2 and NTRK3, in which derepression was unmasked by STZ-induced hyperglycemia, and PAMR1 and MYCL1, in which derepression was present under basal conditions and made more severe by hyperglycemia. In general, a transcriptomic pat- tern was evident in the DACH1 knockdown podocytes that was similar to that in the podocyte-specific Dach1 KO mice. Under basal conditions (Figure 8B), all 4 of the analyzed genes showed modestly increased mRNA levels in DACH1 knockdown podocytes compared with controls, with PAMR1 showing the highest amount of increase and NELL2 the lowest. In control podocytes cultured in media with a high glucose concentration, none of the 4 genes demonstrated increased mRNA expression levels compared with the same cells grown in mannitol (Figure 8B). In DACH1 knockdown podocytes grown in high glucose, however, each of the 4 genes showed severe transcriptional derepression (Figure 8B). Similarly, podocyte viability was reduced only by the combined effects of DACH1 knockdown with hyperglycemia (Supplemental Figure 6). To determine whether transcriptional derepression of DACH1 target genes was a consequence of increased promoter $\mathrm{H} 3 \mathrm{~K} 4 \mathrm{Me} 3$ levels, we performed ChIP-qPCR using an H3K4Me3-specific antibody and primers specific for promoter sequences within NELL2 (Figure 8C) and NTRK3 (Figure 8D) comparing chromatin extracted from DACH1 knockdown and control podocytes with and without hyperglycemia. The results showed increased levels of promoter H3K4Me3 for both NELL2 and NTRK3, which required both DACH1 knockdown and hyperglycemia. Taken together, these findings suggest that transcriptional derepression of DACH1 target genes that is induced by the combination of diminished DACH1 expression and the hyperglycemic condition is caused by augmentation of promoter H3K4Me3 levels.

DACH1-mediated transcriptional repression in podocytes requires DACH1 sequence-specific DNA binding. To substantiate that DACH1-mediated transcriptional repression in podocytes requires direct DNA binding of DACH1 to target promoters, we generated 2 stably transduced human podocyte lines, one carrying a DACH1 expression plasmid and the other a control line carrying the plasmid backbone alone. Western blotting using a DACH1-specific antibody verified overexpression of DACH1 in podocytes carrying the DACH1 expression plasmid (Figure 9A). Next, we cloned the promoter of the DACH1 target gene NELL2 into a lentiviral promoterless luciferase reporter plasmid, which allowed for efficient introduction of the plasmid into podocytes (Figure 9B). A plasmid that contained an identical portion of the NELL2 promoter, except containing a deletion of its DBD $(\triangle \mathrm{DBD})$, was similarly generated. When the control NELL2 promoter plasmid was introduced into podocytes, NELL2 promoter activity was lower in DACH1-overexpressing podocytes compared with controls (Figure 9C). This was true under both basal and high-glucose culture conditions. When the $\triangle \mathrm{DBD}$ plasmid was similarly transduced into podocytes, however, DACH1-mediated transcriptional repression was lost, and luciferase levels were not different between DACH1 overexpressing and control cells. This implies that DACH1-mediated transcriptional repression requires direct promoter binding of DACH1. Furthermore, under high-glucose culture conditions, $\triangle \mathrm{DBD}$ promoter activity was substantially higher than that of the control promoter. This finding corroborates, similarly to what occurred in DACH1 knockdown podocytes, that full DACH1 target-gene derepression requires diminished DACH1-promoter binding combined with hyperglycemia. 


\section{Discussion}

Increasing evidence suggests that genes that mediate human DKD pathogenesis are regulated not only by traditional signaling pathways and transcription factor binding, but also by epigenetic changes, including histone chromatin modifications (20). In the current work, we identify a mechanism by which the tri-methylation of $\mathrm{H} 3 \mathrm{~K} 4$, a modification associated with transcriptional activation, is increased in DACH1 target genes in podocytes in DKD (Figure 10). PTIP is an integral component of the histone methyltransferase complex that also contains catalytic subunits, such as the SET domain proteins MLL2, MLL3, and MLL4, which are responsible for the enzymatic activity of the complex. Although PTIP associates with the MLL proteins, it is not required for their enzymatic activity. What PTIP does do is target the entire H3K4 complex to specific gene promoter sites by interacting with DNAbound transcription factors. The best-characterized example of this is for the transcription factor PAX2, which recruits PTIP- to PAX2-binding sites and in this way, enhances H3K4 tri-methylation and transcriptional activation $(19,24)$. We show that PTIP is also recruited by DACH1 to sequence-specific DACH1 DNA-binding sites, but that this conformation reduces $\mathrm{H} 3 \mathrm{~K} 4 \mathrm{Me} 3$ promoter levels and represses target gene transcription. We do not know whether suppression of methyltransferase activity by DNAbound DACH1-PTIP is caused by diminished recruitment of the MLL-containing portion of the H3K4 complex or by decreased enzymatic activity of the complex once recruited. We propose that when podocyte DACH1 expression is diminished, as in human DKD, PTIP recruitment by DACH1 is lessened and therefore a safeguard that normally limits $\mathrm{H} 3 \mathrm{~K} 4 \mathrm{Me} 3$ activity is lost. In combination with hyperglycemia, this leads to increased promoter H3K4Me3 levels, derepression of multiple podocyte DACH1 target genes, and increased podocyte injury susceptibility.

An important unanswered question is why hyperglycemia is required along with diminished DACH1 expression for full DACH1 target gene derepression. This is evident phenotypically in podocyte-specific Dach1 KO mice, in which podocytes only become injured after the onset of hyperglycemia. Hyperglycemia is known to induce potent epigenetic changes $(20,42)$. An example of the clinical importance of this is metabolic memory, an observation that patients who have poor glycemic control early after diabetes diagnosis, despite good glycemic control for many years afterwards, retain increased risks of DKD and other diabetes-related complications, a phenomenon caused by epigenetic changes (21, 43). Our data suggest that epigenomic changes induced by podocyte loss of DACH1 expression require a second hit for amplification, a role served by hyperglycemia. Further studies will be required to understand the involved mechanisms.

In addition, the severity of podocyte injury induced by hyperglycemia in podocyte-specific Dach1 KO mice was surprising, given that STZ-induced hyperglycemia is typically a mild form of podocyte injury. The phenotype was consistent with severe FSGS induced by hyperglycemia and not DKD. Based on DACH1 IF, podocyte DACH1 expression is nearly completely absent in the podocyte-specific KO mouse, a level of deficiency much more severe than in human DKD. A mouse model in which podocytes have diminished, but not absent, DACH1 expression might be more physiological and perhaps show features more similar to those of human DKD. We also demonstrate that podocyte-specific Dach1 KO mice are exceptionally sensitive to a second form of podocyte injury, ADR nephropathy. This finding suggests that diminished podocyte DACH1 expression may enhance podocyte injury susceptibility not only in DKD, but in other glomerular diseases as well. However, whether the vulnerability of podocyte-specific Dach1 KO mice to ADR is caused by epigenetic modulation of DACH1 target genes has not been shown.

Previous elegant studies reported that sequence-specific DACH1-DNA binding contributed to transcriptional repression by attenuated forkhead signaling $(4,5)$. DACH1 and forkhead family transcription factors were found to have DNA-binding domains that were similar in sequence, causing competitive binding. Whereas DACH1-DNA binding was largely repressive, forkhead-DNA binding activated transcription. Based on this model, reduced levels of podocyte DACH1, as occurs in human $\mathrm{DKD}$, would cause unopposed binding of forkhead proteins to DACH1-binding sites. Interestingly, transcription of multiple forkhead family transcription factors (Foxs1, Foxd2, Foxd1, Fox$d 2 o s$, Foxc1, and Foxj3) was markedly decreased in podocyte-specific Dach1 KO glomeruli under basal conditions compared with controls, with only Foxd1 and Foxd2os remaining downregulated in Dach1 KO glomeruli in early diabetes. We speculate that this downregulation reflects negative feedback aimed at correcting DACH1 target gene derepression. Most likely the DNA-binding competition between DACH1 and forkhead transcription factors occurs in parallel with epigenetic changes mediated by DACH1 once it is DNA bound. These coexisting mechanisms are likely tightly regulated on a cell-type-specific basis.

The seminal observations by Lefevre et al. are in the only previously published study examining the function of PTIP in podocytes (38). They show that podocyte-specific Ptip KO mice under basal condition do not have an immediate glomerular phenotype, but instead develop chronic glomerulopathy at an advanced age. However, they do not report how these mice at a young age would respond to conditions of podocyte stress. Based on the exquisite injury susceptibility of podocyte-specific Dach1 KO mice, we predicted that the Ptip mice might also show high injury vulnerability. Remarkably, microarray analysis performed on glomeruli of young overtly healthy podocyte-specific Ptip KO mice identified only a limited subset of differential gene expression, much of which overlapped with our analysis of podocyte-specific Dach1 KO mice, including in genes not typically thought of as expressed in podocytes, including Prm1 and Pamr1. We investigated the regulation of several overlapping genes, including Nell2 and Nrtk3, both of which are downregulated in Ptip KO glomeruli and upregulated in Dach1 KO glomeruli after diabetes onset. In the same study, NTRK3 was shown to be essential for podocyte function under basal conditions (38). The glomerular function of NELL2, whose expression is highly enriched in podocytes, has not been described. Recently, NELL2's orthologue NELL1 was identified as a podocyte antigen targeted in a subset of primary membranous nephropathy patients (44).

DACH1 target gene derepression disturbs many downstream pathways that likely explain the extraordinary vulnerability of DACH1-deficient podocytes to injury. For example, DACH1 restrains TGF- $\beta$ activity $(8,45,46)$, including diminished 
SMAD2/3 phosphorylation, a pathway whose overactivity is highly implicated in DKD pathogenesis. Consistent with this finding, our RNA-Seq data identified decreased bone morphogenetic protein-7 (Bmp7) mRNA levels in podocyte-specific Dach1 KO glomeruli under both basal conditions and the setting of early diabetes. Preventing loss of BMP7 levels has been shown to protect podocytes from diabetic injury by restraining TGF- $\beta$ signaling $(47,48)$. BMP7-induced SMAD signals block activation and nuclear translocation of TGF- $\beta$-induced SMAD2/3 (49). The mechanism by which loss of podocyte DACH1 leads to diminished BMP7 levels is not certain, but several negative regulators of BMP7, including glypican 3 (GPC3) $(50,51)$, contain promoter DBDs and are substantially upregulated in podocyte-specific Dach1 KO glomeruli both at baseline and in early diabetes. Further studies are required to validate the significance of these observations.

Overall, we show that diminished DACH1 expression in podocytes is a central nidus that leads to derepression of multiple target genes and downstream signals, the consequence of which is exquisite podocyte injury vulnerability. The derepression is caused by diminished recruitment of PTIP by DACH1 to target gene promoters, resulting in an overly active podocyte epigenome. Many potential therapies for DKD have failed in clinical trials because they focus on blocking or augmenting only a single pathway, whereas DKD is a multipathway disease. With this in mind, therapeutic interventions designed to augment podocyte DACH1 activity would correct multiple dysregulated signals simultaneously and therefore should be a priority for DKD drug development and testing.

\section{Methods}

Global and podocyte-specific Dach1 KO mice. The Dach1 mouse strain used was created from ES cell clone EPD0714_4_C11, obtained from the KOMP Repository (www.komp.org) and generated by the Wellcome Trust Sanger Institute (WTSI). Targeting vectors used were generated by the WTSI and the Children's Hospital Oakland Research Institute as part of the Knockout Mouse Project (3U01HG004080). Methods used on the CSD targeted alleles have been previously published (29). Dach1tm1a mice, the nonexpressive form, were resuscitated by the KOMP repository at our request. These mice were on a C57BL/6 genetic background. Dach1tm1a mice were also converted to the conditional allele (Dach1tm1c) by breeding to ubiquitously expressed Flp-recombinase transgenic mice (C57BL/6N-Tg[CAG-Flpo]1Afst/Mmucd) that were purchased from the Mutant Mouse Resource and Research Center (MMRRC). Resultant Dach1tm1c mice were backcrossed twice to WT C57BL/6 mice to ensure removal of the Flp-recombinase transgene. Dach1tm1c mice were then bred to transgenic mice expressing cre recombinase driven by the podocyte-specific podocin promoter (podocin-cre) to generate podocyte-specific Dach1 KO mice (Dach1tm1d). Mice were genotyped using the following primers: Dach1tm1a, PreCre (amplicon size 541 bp): CSD-neoF: GGGATCTCATGCTGGAGTTCTTCG and CSD-Dach1-ttR: AACAATTCTTGTCCTTCACGTGCCC; Dach1tm1c and Dach1tm1d, PostFlp (amplicon size 631 bp) and WT (amplicon size 496 bp): CSD-Dach1-F: CTCCTGAAGATGAGGAGCTCACCCC and CSD-Dach1-ttR. Podocin-cre transgenic mice on a C57BL/6 background were previously purchased from The Jackson Laboratory and genotyped using their recommended primers.

Inducible podocyte-specific Dach1 transgenic mice. V5-tagged murine Dach1 cDNA was synthesized and cloned into the XmaI-HindIII site of pTRE-Tight (Takara Bio USA) by Genscript. The resulting vector, pTRE-
Dach1, was linearized and then used for pronuclear injections (FVB/N strain) performed by the Mouse Genetics and Gene Targeting Core Facility at Icahn School of Medicine at Mount Sinai. pTRE-Dach1 transgenic mice were genotyped using the following primers: S2-GCAAGAGACAGCATCGAC, A2-AATCGGTTTGCCGTACAT and S3-CTAACTGgGCATGgACAA, A3-GgAATCGgtTTGCCGTACA. Podocin-rtTA mice, on an $\mathrm{FVB} / \mathrm{N}$ background, were originally purchased from The Jackson Laboratory and genotyped using their recommended primers.

Diabetes mouse models. For induction of type I diabetes with STZ, mice at 8 weeks of age were injected over 5 consecutive days with lowdose STZ (50 $\mu \mathrm{g} / \mathrm{g} / \mathrm{d}$ intraperitoneally; Sigma-Aldrich). Blood glucose levels were monitored weekly by glucometer readings. Diabetes was confirmed by fasting blood glucose levels greater than $300 \mathrm{mg} / \mathrm{dl}$. The age-matched littermates injected with vehicle alone served as nondiabetic controls. Urine samples were collected on a weekly basis. Marked proteinuria and signs of overall debility in many podocyte-specific Dach1 KO mice became evident several weeks after onset of diabetes. Mice were sacrificed 1 month after diabetes onset. OVE26 mice (FVB/N background) were originally obtained from Jackson Laboratories and genotyped according to their protocol. Blood glucose levels were monitored every other week. All animals in all experimental groups were fed DOX hyclate-impregnated $(625 \mathrm{mg} / \mathrm{kg})$ rodent chow (Envigo) starting at weaning age of 3 weeks that was continued until euthanasia. By spot urine measurements, we noted diminished proteinuria in OVE26 mice that also carried pTRE-Dach1 and podocin-rtTA transgenes, starting at approximately 8 weeks of age. At 14 weeks of age, mice were moved to metabolic cages to collect a 24-hour urine sample, after which animals were euthanized and kidney tissues harvested.

ADR nephropathy. Baseline urine was collected for podocyte-specific Dach1 KO mice and control littermates. All mice were administered ADR $(20 \mathrm{mg} / \mathrm{kg})$ intravenously by retroorbital injection. Urine was collected weekly and heavy proteinuria and overall signs of severe debility noted in almost all Dach1 KO mice by 1 week after ADR injection. All surviving mice were euthanized at 2 weeks after ADR treatment and kidneys harvested for analysis.

Glomerular morphometry. Quantification of foot-process effacement and GBM width was performed using Image J software (NIH) on digitized TEM images, as previously described (25). For quantification of GBM thickness, an average of 10 measurements from each of 3 different glomeruli (30 total measurements) was taken per mouse (from podocyte to endothelial cell membranes) at sites where the GBM was displayed in the best cross section. The same glomeruli were scored for degree of podocyte effacement. The Cavalieri principle was used to measure glomerular volume $(52,53)$. Ten glomeruli were measured per kidney. An average of 905.3 points were counted per kidney. The volumes of individual glomerular components were assessed as previously described $(54,55)$.

Calculation of podocyte number. The number of WT1-positive cells per glomerular tuft was determined for glomeruli where the macula densa could be clearly visualized, which ensured that all glomeruli were analyzed from a similar level of sectioning. Glomerular area was calculated using ImageJ software.

Mutagenic screen. The mutagenic screen was carried out as previously described (17). The HIV-1 plasmid used for the screen was the replication-incompetent pNL4-3 $\Delta g a g$-pol, which expresses all of the HIV regulatory proteins, but not the HIV structural proteins gag and pol (25). pNL4-3 $\Delta$ gag-pol expression in podocytes promotes anchorage-independent growth, a response that depends on the HIV nef gene (25). 
RNA-Seq. Total RNA was extracted from isolated glomeruli by using the RNeasy Mini Kit (QIAGEN) according to the manufacturer's protocol. RNA concentrations were quantified using a Nano-drop Spectrophotometer at a wavelength of $260 \mathrm{~nm}$. RNA samples at a concentration of 100-200 ng/ $\mu$ g were provided to the Genomics Resources Core Facility at Weill Cornell Medicine for mRNA sequencing. Bioinformatic analysis was performed as follows. After read quality control, mapping, and normalization steps on the raw sequencing reads, the expression data were compared between glomeruli of control and podocyte-specific Dach1 KO mice at baseline and also early after induction of diabetes with STZ, to identify differentially expressed genes (DEGs) with a $P$ value less than 0.05 using the limma test. The DEGs were then subjected to enrichment analysis for canonical pathway and gene ontology. RNA-Seq data were deposited in the NCBI's Gene Expression Omnibus database (GEO GSE168676). DEGs were overlapped with a published microarray data set from podocyte-specific Ptip KO mice (38). In the microarray of podocyte-specific Ptip KO mice, those DEGs that were named only by RIKEN cDNA number and were not reported as being expressed in the RNA-Seq data from the podocyte-specific Dach1 KO mice were excluded from the analysis.

In silico promoter analysis. Using TRANSFAC software (56), we scanned the promoters of all mouse genes in the region from -2500 to +500 using the previously reported DACH1 positional weight matrix, allowing for both exact and single-nucleotide mismatches. The promoter sequence data, derived from the NCBI, for all 20 genes in the Dach1-Ptip overlap gene set were reviewed manually to confirm, identify, and localize all DBDs.

ChIP-qPCR. ChIP-qPCR was performed using a ChIP kit (Cell Signaling Technologies, 9003) per the manufacturer's protocol. The number of cells utilized per experiment was as follows: $1.5 \times 10^{7}$ for transfected 293T cells and $6 \times 10^{7}$ for transduced human podocytes. Sonication conditions were optimized to generate chromatin fragments of between 250 and 1000 base pairs. Duration of sonication was reduced for transient transfection experiments involving chromatin from plasmid DNA. Purified DNA was subjected to real-time PCR using primer sequences shown in Supplemental Figure 7. Results are presented as normalized against diluted input DNA.

Antibodies. The following antibodies were used: PTIP (Millipore Sigma, catalog ABE1877), H3K4Me3 (Cell Signaling Technologies, catalog 9751), DACH1 (MilliporeSigma, catalog HPA012672, IF), DACH1 (Proteintech, catalog 10914-1-AP, WB), NELL2 (MilliporeSigma, catalog HPA035715), FLAG tag (M2 antibody, Millipore Sigma, catalog F1804), V5 tag (MilliporeSigma, catalog V8137), GAPDH (Cell Signaling Technologies, clone 14C10), WT1 (Abcam, catalog ab89901), and Ki67 (Cell Signaling Technologies, catalog 12202).

Co-IP and DBD-multimeric reporter assay. Plates of 293T cells were individually transfected with DACH1-V5, PTIP-FLAG (Genscript, catalog OHU18275D), or FLAG-RAVER expression plasmids. Total cellular lysates were harvested in RIPA buffer, combined at equal concentrations, and then incubated overnight with anti-FLAG M2 magnetic beads (MilliporeSigma, M8823). Beads were washed with RIPA buffer $\times 3$ and then boiled. Western blotting was performed on the resulting eluates and on input lysates using antibodies to FLAG and V5. Six copies of the DRE (TATTTATTTGTATTCATTTATTTAATTGTATTGT) were synthesized as a multimer (synthesized and cloned by Genscript) and then cloned into the MluI and BglII sites of vector pGL3-control (Promega). The resulting plasmid is similar to one previously described (5). Full- length DACH1-V5 and DACH1- $\triangle \mathrm{DBD}-\mathrm{V} 5$, carrying a 297-nucleotide deletion that corresponds to its box-N domain, were each cloned into pcDNA3.1 (synthesized and cloned by Genscript). The DRE multimeric pGL3-control vector or pGL3-control vector alone were cotransfected with a DACH1-V5 expression plasmid (either full-length or $\triangle \mathrm{DBD}$ ) into 293T cells and luciferase activity measured using Dual-Glo Luciferase Assay System (Promega) per the manufacturer's protocol.

qPCR. Total RNA was extracted using the RNeasy Mini Kit. Two micrograms of total RNA were subjected to reverse transcription to generate cDNA using Superscript IV reverse transcriptase (Thermo Fisher Scientific). Diluted cDNA was amplified in triplicate using SYBR Green Master Mix (Bio-Rad) using primers outlined in Supplemental Figure 7. Light cycler software was used to determine crossing points, and results were normalized to housekeeping gene $18 \mathrm{~S}$ rRNA.

Digital image analysis. Images of glomeruli of immunostained human kidney tissue were taken at $\times 40$ magnification at the Icahn School of Medicine at Mount Sinai Microscopy Shared Resource Facility. A fixed exposure time was set based on the fluorescence intensity of the most strongly fluorescent glomeruli. To determine intensity of staining, we drew a region of interest around each glomerulus and then measured the mean luminosity of the region using the software analysis program MetaMorph. A similar approach was used to determine the mean luminosity of DAPI and WT1.

Histology, IF, and electron microscopy of mouse tissue. All kidneys were perfused in vivo with $4 \%$ PFA. For histological analysis, harvested kidneys were then fixed overnight in PFA and embedded in paraffin. Sections were cut at $2 \mu \mathrm{m}$ and stained with periodic acid-Schiff (PAS). For IF, kidneys were fixed in PFA for 4 hours, transferred to $18 \%$ sucrose overnight, and then flash-frozen in OCT medium. For electron microscopy, kidneys were fixed in $2.5 \%$ glutaraldehyde and embedded in epoxy resin using standard techniques. TEM was performed using a JEOL 1011 electron microscope and SEM with a Hitachi S430 field emission scanning electron microscope.

Podocyte cell culture. Generation and propagation of established conditionally immortalized podocyte cell lines has been described previously (57). Briefly, podocytes were propagated on type I collagen-coated dishes at the permissive temperature $\left(33^{\circ} \mathrm{C}\right)$ in RPMI supplemented with $10 \%$ FBS and $1 \%$ (vol/vol) insulin-transferrin-selenium (Thermo Fisher Scientific). To allow for differentiation, cells were shifted to the nonpermissive temperature $\left(37^{\circ} \mathrm{C}\right)$.

DACH1 knockdown podocytes. For DACH1 knockdown, lentiviral expression vectors (pGFP-C-shLenti) carrying shRNAs targeting DACH1 were purchased from Origene (TL313572). A noneffective 29-mer scrambled shRNA cassette cloned in the same vector was also purchased from Origene. Lentiviral preparations and infections were performed as previously described (25). Infections were done at the permissive temperature in human podocytes, and then stable cell lines were established by selection with puromycin. Stable DACH1 knockdown lines were grown at $37^{\circ} \mathrm{C}$ for at least 1 week prior to use in experiments. Cellular viability was assessed using a kit (Promega, G4000) according to the manufacturer's protocol.

DACH1 overexpression podocytes and NELL2 promoter reporter assays. For overexpression of DACH1, human DACH1 cDNA was subcloned into the NotI-XbaI restriction sites of the lentiviral vector PSF-Lenti (MilliporeSigma). Lentiviral preparations and infections were performed as previously described for both PSF-Lenti-DACH1 and PSF-Lenti vector alone (25). Infections were carried out at the per- 
missive temperature in human podocytes, and then stable cell lines were selected with puromycin. The promoter of NELL2, including the 2003 bp immediately upstream of its ATG start, was synthesized and cloned into the SalI-BamH1 sites of the promoterless luciferase reporter lentiviral vector LVR-1048 (Cellomics Technology). DNA synthesis and cloning were performed by Genscript. The single DBD contained in the NELL2 promoter (13 base pairs from -1166 to -1199 upstream of the ATG site) was deleted and then cloned similarly into LVR-1048 to generate the NELL2 $\triangle \mathrm{DBD}$ plasmid (mutagenesis and cloning performed by Genscript). Viral particles containing the full-length NELL2 or $\triangle \mathrm{DBD}$ plasmid were generated as previously described, after which the functional titer of each was measured. Human podocytes that had been stably transduced with PSF-Lenti or PSF-Lenti-DACH1 were then transduced with NELL2 or $\triangle \mathrm{DBD}$ promoter luciferase viral particles at the permissive temperature, after which cells were immediately shifted to $37^{\circ} \mathrm{C}$ for 1 week. Each experiment involved freshly infected cells. Luciferase activity was measured using the Dual-Glo Luciferase Assay System (Promega) per the manufacturer's protocol.

Nephroseq. The Nephroseq database is accessible at https://www. nephroseq.com. Raw microarray data for individual patients were extracted from Nephroseq and converted to a nonlogarithmic scale.

Statistics. All experiments were repeated 3 or more times. All data are represented as mean \pm SEM. Statistical significance was determined by utilizing 2-tailed Student's $t$ test or by 1-way ANOVA and Tukey's post hoc test. A $P$ value of less than 0.05 was considered significant. Prism software (GraphPad) was used for comparison between groups.

Study approvals. Animal studies were performed in accordance with the guidelines of and approved by the Institutional Animal Care and Use Committee at the Icahn School of Medicine at Mount Sinai and followed NIH guidelines. All human material was obtained from an IRB-approved protocol from the Icahn School of Medicine at Mount Sinai to collect extra kidney tissue from patients who underwent a medically indicated nephrectomy for renal cell cancer. Noncancerous kidney material was collected and a diagnosis of DKD was made based on histological and clinical parameters. All nephrectomies were clinically indicated, and only extra tissue not required for diagnostic purposes was permitted for research use; therefore, no patient informed consent was required.

\section{Author contributions}

AC, JL, MA, JMB, BZ, VDD, and LK performed the experiments, data acquisition, and data analysis. Data analysis was also performed by ZY, SJ, TD, OES, NP, and WZ. AC, PC, EUA, KNC, MM, SC, HW, KZ, ZL, BM, JCH, VDD, KS, and LK conceptualized and designed the studies. All authors participated in drafting and critical appraisal of the manuscript.

\section{Acknowledgments}

This work was supported by National Institute of Diabetes and Digestive and Kidney Diseases, NIH, grant 1R01DK121978 (to LK) and NIH grant 1R01DK104712 (to LK).

Address correspondence to: Lewis Kaufman, Icahn School of Medicine at Mount Sinai, Annenberg Building, Room 23-68D, One Gustave L Levy Place, New York, New York 10029, USA. Phone: 646.245.2294; Email: lewis.kaufman@mssm.edu.
1. Davis RJ, et al. Dach1 mutant mice bear no gross abnormalities in eye, limb, and brain development and exhibit postnatal lethality. Mol Cell Biol. 2001;21(5):1484-1490.

2. Wu K, et al. Endogenous Dach1 in cancer. Oncoscience. 2015;2(10):803-804.

3. Kong $\mathrm{D}$, et al. The retinal determination gene network: from developmental regulator to cancer therapeutic target. Oncotarget. 2016;7(31):50755-50765.

4. Zhou J, et al. Attenuation of Forkhead signaling by the retinal determination factor DACH1. Proc Natl Acad Sci U S A. 2010;107(15):6864-6869.

5 . Zhou J, et al. Transcription elongation regulator 1 is a co-integrator of the cell fate determination factor Dachshund homolog 1. J Biol Chem. 2010;285(51):40342-40350.

6. Li X, et al. Eya protein phosphatase activity regulates Six1-Dach-Eya transcriptional effects in mammalian organogenesis. Nature. 2003;426(6964):247-254.

7. $\mathrm{Wu} \mathrm{K}$, et al. Cell fate determination factor DACH1 inhibits c-Jun-induced contact-independent growth. Mol Biol Cell. 2007;18(3):755-767.

8. Wu K, et al. DACH1 inhibits transforming growth factor-beta signaling through binding Smad4.J Biol Chem. 2003;278(51):51673-51684.

9. $\mathrm{Wu} \mathrm{K}$, et al. DACH1 is a cell fate determination factor that inhibits cyclin D1 and breast tumor growth. Mol Cell Biol. 2006;26(19):7116-7129.

10. Liu QQ, et al. Decreased DACH1 expression in glomerulopathy is associated with disease progression and severity. Oncotarget. 2016;7(52):86547-86560.
11. Endlich N, et al. The transcription factor Dach1 is essential for podocyte function. J Cell Mol Med. 2018;22(5):2656-2669.

12. Kottgen A, et al. New loci associated with kidney function and chronic kidney disease. Nat Genet. 2010;42(5):376-384.

13. Boger CA, et al. Association of eGFR-related loci identified by GWAS with incident CKD and ESRD. PLoS Genet. 2011;7(9):e1002292.

14. Rinschen MM, et al. A multi-layered quantitative in vivo expression atlas of the podocyte unravels kidney disease candidate genes. Cell Rep. 2018;23(8):2495-2508.

15. No authors listed. USRDS: the United States Renal data system. Am J Kidney Dis. 2003;42(6 Suppl 5):1-230.

16. Gregg EW, et al. Changes in diabetes-related complications in the United States, 1990-2010. N Engl J Med. 2014;370(16):1514-1523.

17. Potla U, et al. Podocyte-specific RAP1GAP expression contributes to focal segmental glomerulosclerosis-associated glomerular injury. J Clin Invest. 2014;124(4):1757-1769.

18. Cho YW, et al. PTIP associates with MLL3and MLL4-containing histone $\mathrm{H} 3$ lysine 4 methyltransferase complex. JBiol Chem. 2007;282(28):20395-20406.

19. Munoz IM, Rouse J. Control of histone methylation and genome stability by PTIP. EMBO Rep. 2009;10(3):239-245.

20. Kato M, Natarajan R. Epigenetics and epigenomics in diabetic kidney disease and metabolic memory. Nat Rev Nephrol. 2019;15(6):327-345.
21. Park J, et al. Functional methylome analysis of human diabetic kidney disease. JCI Insight. 2019;4(11):128886.

22. Lin CL, et al. A KDM6A-KLF10 reinforcing feedback mechanism aggravates diabetic podocyte dysfunction. EMBO Mol Med. 2019;11(5):e9828.

23. Majumder S, et al. Shifts in podocyte histone $\mathrm{H} 3 \mathrm{~K} 27 \mathrm{me} 3$ regulate mouse and human glomerular disease. J Clin Invest. 2018;128(1):483-499.

24. Patel SR, et al. The BRCT-domain containing protein PTIP links PAX2 to a histone H3, lysine 4 methyltransferase complex. Dev Cell. 2007;13(4):580-592.

25. Husain M, et al. HIV-1 Nef induces proliferation and anchorage-independent growth in podocytes. J Am Soc Nephrol. 2002;13(7):1806-1815.

26. Gharavi AG, et al. Mapping a locus for susceptibility to HIV-1-associated nephropathy to mouse chromosome 3. Proc Natl Acad Sci U S A. 2004;101(8):2488-2493.

27. Hodgin JB, et al. A molecular profile of focal segmental glomerulosclerosis from formalin-fixed, paraffin-embedded tissue. Am J Pathol. 2010;177(4):1674-1686

28. Pan Y, et al. Dissection of glomerular transcriptional profile in patients with diabetic nephropathy: SRGAP2a protects podocyte structure and function. Diabetes. 2018;67(4):717-730.

29. Skarnes WC, et al. A conditional knockout resource for the genome-wide study of mouse gene function. Nature. 2011;474(7351):337-342.

30. Schild R, et al. Double homozygous missense mutations in DACH1 and BMP4 in a patient 
with bilateral cystic renal dysplasia. Nephrol Dial Transplant. 2013;28(1):227-232.

31. Wharram BL, et al. Podocyte depletion causes glomerulosclerosis: diphtheria toxin-induced podocyte depletion in rats expressing human diphtheria toxin receptor transgene. J Am Soc Nephrol. 2005;16(10):2941-2952.

32. Shirata N, et al. Glomerulosclerosis induced by deficiency of membrane-associated guanylate kinase inverted 2 in kidney podocytes. J Am Soc Nephrol. 2017;28(9):2654-2669.

33. Hakroush S, et al. Extensive podocyte loss triggers a rapid parietal epithelial cell response. J Am Soc Nephrol. 2014;25(5):927-938.

34. Reiniger N, et al. Deletion of the receptor for advanced glycation end products reduces glomerulosclerosis and preserves renal function in the diabetic OVE26 mouse. Diabetes. 2010;59(8):2043-2054.

35. Cho C, et al. Haploinsufficiency of protamine-1 or -2 causes infertility in mice. Nat Genet. 2001;28(1):82-86.

36. Okada Y, et al. Histone demethylase JHDM2A is critical for Tnp1 and Prm1 transcription and spermatogenesis. Nature. 2007;450(7166):119-123.

37. Schwab KR, et al. Arrested spermatogenesis and evidence for DNA damage in PTIP mutant testes. Dev Biol. 2013;373(1):64-71.

38. Lefevre GM, et al. Altering a histone H3K4 methylation pathway in glomerular podocytes promotes a chronic disease phenotype. PLoS Genet. 2010;6(10):e1001142.

39. Hodgin JB, et al. Identification of cross-species shared transcriptional networks of diabetic nephropathy in human and mouse glomeruli. Diabetes. 2013;62(1):299-308.

40. Woroniecka KI, et al. Transcriptome analysis of human diabetic kidney disease. Diabetes. 2011;60(9):2354-2369.

41. Kim SS, et al. Structure of the retinal determination protein Dachshund reveals a DNA binding motif. Structure. 2002;10(6):787-795.

42. Reddy MA, et al. Epigenetic mechanisms in diabetic complications and metabolic memory. Diabetologia. 2015;58(3):443-455.

43. de Boer IH, et al. Long-term renal outcomes of patients with type 1 diabetes mellitus and microalbuminuria: an analysis of the Diabetes Control and Complications Trial/Epidemiology of Diabetes Interventions and Complications cohort. Arch Intern Med. 2011;171(5):412-420.

44. Sethi S, et al. Neural epidermal growth factor-like 1 protein (NELL-1) associated membranous nephropathy. Kidney Int. 2020;97(1):163-174.

45. Jiao X, et al. Dachshund depletion disrupts mammary gland development and diverts the composition of the mammary gland progenitor pool. Stem Cell Reports. 2019;12(1):135-151.

46. Zhu H, et al. Epigenetic silencing of DACH1 induces loss of transforming growth factor- $\beta 1$ antiproliferative response in human hepatocellular carcinoma. Hepatology. 2013;58(6):2012-2022.

47. Wang S, et al. Renal bone morphogenetic protein-7 protects against diabetic nephropathy. $J$ Am Soc Nephrol. 2006;17(9):2504-2512.

48. Mitu GM, et al. BMP7 is a podocyte survival factor and rescues podocytes from diabetic injury. $\mathrm{Am} \mathrm{J}$
Physiol Renal Physiol. 2007;293(5):F1641-F1648.

49. Wang S, Hirschberg R. Bone morphogenetic protein-7 signals opposing transforming growth factor beta in mesangial cells. J Biol Chem. 2004;279(22):23200-23206.

50. Chang C. Agonists and antagonists of TGF- $\beta$ family ligands. Cold Spring Harb Perspect Biol. 2016;8(8):a021923.

51. Midorikawa Y, et al. Glypican-3, overexpressed in hepatocellular carcinoma, modulates FGF2 and BMP-7 signaling. Int J Cancer. 2003;103(4):455-465.

52. Basgen JM, Sobin C. Early chronic low-level lead exposure produces glomerular hypertrophy in young C57BL/6J mice. Toxicol Lett. 2014;225(1):48-56.

53. Gundersen HJ, Jensen EB. The efficiency of systematic sampling in stereology and its prediction. JMicrosc. 1987;147(Pt 3):229-263.

54. Wei C, et al. SHROOM3-FYN interaction regulates nephrin phosphorylation and affects albuminuria in allografts. J Am Soc Nephrol. 2018;29(11):2641-2657.

55. Steffes MW, et al. Amelioration of mesangial volume and surface alterations following islet transplantation in diabetic rats. Diabetes. 1980;29(7):509-515.

56. Matys V, et al. TRANSFAC: transcriptional regulation, from patterns to profiles. Nucleic Acids Res. 2003;31(1):374-378.

57. Saleem MA, et al. A conditionally immortalized human podocyte cell line demonstrating nephrin and podocin expression. J Am Soc Nephrol. 2002;13(3):630-638. 